\title{
Spatiotemporal Profile and Morphological Changes of NG2 Glia in the CA1 Region of the Rat Hippocampus after Transient Forebrain Ischemia
}

\author{
Xuyan Jin ${ }^{1,2}$, Tae-Ryong Riew ${ }^{1}$, Soojin Kim ${ }^{1}$, Hong Lim Kim ${ }^{3}$ and Mun-Yong Lee ${ }^{1,2 *}$ \\ ${ }^{1}$ Department of Anatomy, Catholic Neuroscience Institute, College of Medicine, The Catholic University of Korea, ${ }^{2}$ Department \\ of Biomedicine and Health Sciences, College of Medicine, The Catholic University of Korea, ${ }^{3}$ Integrative Research Support \\ Center, Laboratory of Electron Microscope, College of Medicine, The Catholic University of Korea, Seoul 06591, Korea
}

\begin{abstract}
Neuron-glial antigen-2 (NG2) glia undergo proliferation and morphological changes following brain insults. Here, we show that NG2 glia is activated in a characteristic time- and layer-specific manner in the ischemia-vulnerable CA1 region of the rat hippocampus. Resting NG2 glia of the pyramidal cell layer (somatic region) shared morphological features with those of the neighboring dendritic stratum radiatum. During the postischemic period, reactive NG2 glia of the pyramidal cell layer exhibited shortened, scarcely branched processes, while those of the stratum radiatum had multiple branching processes with their arborization being almost indiscernible 7 14 days after reperfusion. Immunoelectron microscopy demonstrated that NG2 immunoreactivity was specifically associated with the plasma membrane and the adjacent extracellular matrix of NG2 glia in the stratum radiatum at 14 days. NG2 glia also exhibited differences in their numbers and proliferation profiles in the two examined hippocampal strata after ischemia. In addition, induced NG2 expression in activated microglia/macrophages exhibited a characteristic strata-dependent pattern in the ischemic CA1 hippocampus. NG2 induction was prominent in macrophage-like phenotypes which were predominantly localized in the pyramidal cell layer, compared with activated stellate microglial cells in the stratum radiatum. Thus, our data demonstrate that activation of NG2 glia and the induction of NG2 expression in activated microglia/macrophages occur in a distinct time- and layer-specific manner in the ischemic CA1 hippocampus. These characteristic profiles of reactive NG2 glia could be secondary to the degeneration processes occurring in the cell bodies or dendritic domains of hippocampal CA1 pyramidal neurons after ischemic insults.
\end{abstract}

Key words: Hippocampus, Microglia, NG2 glia, Pyramidal cell layer, Stratum radiatum

\section{INTRODUCTION}

Neuron-glial antigen-2 (NG2) glia, characterized by the expression of chondroitin sulphate proteoglycan 4 on their surface, were first described as oligodendrocyte precursor cells but are now recognized as a fourth neuroglial cell type in the central nervous system (CNS) [1-4]. NG2 glia are evenly distributed within the

Received December 3,2019, Revised January 17,2020,

Accepted January 18, 2020

* To whom correspondence should be addressed.

TEL: 82-2-2258-7261, FAX: 82-2-536-3110

e-mail:munylee@catholic.ac.kr grey and white matter of the adult CNS, representing a highly heterogeneous population with diverse properties and functions [4-6]. Previously published findings suggest an active and functional role of NG2 glia in the adult CNS in addition to their role as progenitors for oligodendrocytes.

It is well established that NG2 glia undergo proliferation and morphological changes in response to various CNS insults, including demyelinating diseases [4,7-11], stab wounds $[12,13]$, ischemia $[14,15]$, and spinal cord injury [8]. In addition, our recent study shows that activation of NG2 glia in response to the mitochondrial toxin 3-nitropropionic acid is characterized by progressive morphological changes, higher proliferation rates, and more intense immunostaining for the proteoglycan NG2 [16]. In particular, our 
data provide evidence for the link between the transformation of NG2 glia to their reactive form and microglial activation/recruitment in response to brain insults. In addition to its constitutive expression in NG2 glia, NG2 is also induced in activated microglia and infiltrating macrophages. This has been observed in various CNS insults [16-24], although the specific roles of NG2 induction in these cells are still unknown. NG2 is also expressed in vascular mural cells, wherein it is upregulated during structural remodeling under pathological conditions [16,25]. Despite the heterogeneous populations of NG2-expressing cells in the injured CNS, NG2 glia are characterized only by their expression of one specific antigen the proteoglycan NG2 [4]. Thus, NG2 glia and NG2-positive microglia/macrophages should be distinguished using sections double-labeled for NG2 and microglial markers and carefully paying attention to the morphological characteristics of both glial cell types.

Transient forebrain ischemia causes the selective and delayed neuronal death of hippocampal CA1 neurons in the rat brain, which in turn evokes neuroglial responses [26-30]. In particular, microglial activation varies within the different CA1 sub-layers of the ischemic hippocampus: early accumulation of activated microglia and macrophages occurs in the pyramidal cell layer, the site of intense neural damage, while in the stratum radiatum with its dendritic degeneration, microglia transform initially into elongated rod cells, whereas amoeboid-like macrophages invade the tissue later with the still persisting rod cells being the predominant microglial cell type [31-33]. Interestingly, Ong and Levine [34] show that the densities of NG2 glia in somatic regions (pyramidal cell and granule cell layers) are lower than those in the neighboring dendritic subfields, despite the ubiquitous distribution of NG2 glia throughout the brain. In light of these results, we hypothesized that nature and time course of the appearance of reactive NG2 glia after ischemic insults are distinct in the different hippocampal layers. Although activation and morphological changes of NG2 glia in the CA1 region of the rat hippocampus have been demonstrated in response to hypoxia/ischemia [15], only a few studies examined the effects of transient cerebral ischemia on reactive NG2 glia within different CA1 sub-layers and their relationship with microglial activation.

In the present study, we investigated the spatiotemporal distribution and morphological features of NG2-expressing cells, i.e., NG2 glia and NG2-positive microglia/macrophages in the CA1 hippocampus after transient forebrain ischemia. In particular, we focused our attention on the NG2 glia within different sub-layers (e.g., pyramidal cell layer and stratum radiatum) of the CA1 hippocampus using confocal imaging and correlative light and electron microscopy.

\section{MATERIALS AND METHODS}

\section{Animal preparation}

All experimental protocols and animal care provisions were in accordance with the Laboratory Animals Welfare Act, the Guide for the Care and Use of Laboratory Animals, and the Guidelines and Policies for Rodent Survival Surgery provided by the IACUC (Institutional Animal Care and Use Committee) at the College of Medicine of The Catholic University of Korea (Approval number: CUMS-2017-0321-05). IACUC and the Department of Laboratory Animals (DOLA) in the Catholic University of Korea, Songeui Campus accredited the Korea Excellence Animal Laboratory Facility from the Korea Food and Drug Administration in 2017 and acquired full Association for Assessment and Accreditation of Laboratory Animal Care (AAALAC) International accreditation in 2018. All efforts were made to minimize animal suffering and to reduce the number of animals used.

Adult male Sprague-Dawley rats (250 300 g, OrientBio, Seongnam, Republic of Korea) were used in this study. The animals were housed in groups of three per cage in a controlled environment at a constant temperature $\left(22 \pm 5^{\circ} \mathrm{C}\right)$ and humidity $(50 \pm 10 \%)$ with food (gamma ray-sterilized diet) and water (autoclaved tap water) available ad libitum. Transient forebrain ischemia was induced by the four-vessel occlusion and reperfusion method described by Pulsinelli and Brierley [35], with minor modifications [36]. Briefly, the vertebral arteries were electrocauterized and cut to stop circulation in these vessels. After $24 \mathrm{~h}$, both common carotid arteries were occluded for $10 \mathrm{~min}$ with miniature aneurismal clips. Body temperatures (measured rectally) were maintained at $37.5 \pm 0.3^{\circ} \mathrm{C}$ with a heating lamp during and after ischemia. Sham-operated rats, with cauterized vertebral arteries and ligatures placed around the carotid arteries, were used as controls. The following inclusion criteria were applied: (1) Only those animals lacking a righting reflex after vascular occlusion were classified as demonstrating ischemia and (2) The reduction or absence of neurons in the CA1 region was verified using Nissl-stained sections. No animal convulsed or died following reperfusion or sham operation. After the surgery, animals were monitored twice daily to determine their health and activity levels, i.e., we examined their behavioral changes (activity and food intake), body weight, and body temperature. The overall mortality rate of ischemic rats was lower than $5 \%$, and all sham-operated rats survived after the operation. Most of the deaths occurred during or shortly after the ischemic procedure, and the leading cause of death appeared to be related to cardiac arrest. However, none of the rats were eliminated from the analysis in the days following the ischemic insult owing to sickness or death, and we did not observe any prominent changes in the behavior or 
body weight of the rats during the experiments.

Animals were sacrificed at $3,7,14$, or 28 days after reperfusion ( $\mathrm{n}=5$ rats/time point for ischemic group; $\mathrm{n}=3 /$ time point for the sham-operation group). The animals were transcardially perfused with $4 \%$ paraformaldehyde in $0.1 \mathrm{M}$ phosphate buffer (PB, $\mathrm{pH} 7.4$ ) after anesthesia using $10 \%$ chloral hydrate $(4 \mathrm{~mL} / \mathrm{kg}$ i.p.). The brain tissues were equilibrated with $30 \%$ sucrose in $0.1 \mathrm{M} \mathrm{PB}$ and frozen and stored at $-70^{\circ} \mathrm{C}$ for light microscopic study.

\section{Immunohistochemistry}

For triple fluorescence immunohistochemistry, free-floating sections ( $25 \mu \mathrm{m}$ thick) were blocked in blocking buffer $(0.2 \%$ gelatine, $1 \%$ bovine serum albumin, and $0.05 \%$ saponin) and then incubated at $4^{\circ} \mathrm{C}$ overnight with a mixture of mouse monoclonal antibody against NG2 (1:200; Millipore, Temecula, CA, USA), goat polyclonal antibody against ionized calcium-binding adaptor molecule 1 (Iba1; 1:500; Abcam, Cambridge, UK), and rabbit polyclonal antibody targeting Ki67 (1:1000; Novocastra Laboratories, Ltd., Newcastle upon Tyne, UK). In some experiments, double-labeling was performed using a mix of rabbit polyclonal antibody against NG2 (1:500; Millipore) and one of the following antibodies: goat polyclonal antibody against Ibal (1:500; Abcam) or mouse monoclonal antibody against the neuronal nuclear antigen (NeuN; 1:400; Millipore), glial fibrillary acidic protein (GFAP; 1:700; Millipore), or adenomatous polyposis coli (APC; also called CC1; 1:100; Sigma-Aldrich, St. Louis, MO, USA). This triple- or double-labeling was followed by a 2 -h incubation with appropriate secondary antibodies, as follows: Cy3-conjugated goat anti-mouse antibody (1:2000; Jackson ImmunoResearch, West Grove, PA, USA), Cy3-conjugated donkey anti-goat antibody (1:2000; Jackson ImmunoResearch), Alexa Fluor 488-tagged goat anti-rabbit antibody (1:300; Thermo Fisher, Waltham, MA, USA), Alexa Fluor 488-tagged donkey anti-mouse antibody (1:300; Thermo Fisher), or Alexa Fluor 647-tagged donkey anti-rabbit antibody (1:300; Thermo Fisher). Some sections were also incubated at $4^{\circ} \mathrm{C}$ overnight with mouse monoclonal antibody against microtubule-associated protein 2 (MAP2; 1:200; Sigma-Aldrich). Counterstaining of cell nuclei was carried out with 4', 6-diamidino2'-phenylindole (DAPI; 1:2000; Roche, Mannheim, Germany) for 10 min. Negative staining controls for double or triple immunofluorescence were performed by omission of the primary or secondary antibodies. In addition, we compared the results of double- or triple-labeling procedures with those from single- and double-labeling experiments for all combinations of antibodies to ensure a clear interpretation and to detect cross-reactivities. Slides were viewed under a confocal microscope (LSM 700; Carl Zeiss Co., Ltd., Oberkochen, Germany) equipped with four lasers (Diode 405, Argon 488, HeNe
555, and HeNe 639) under constant viewing conditions. For 3D reconstruction, NG2 and Iba1 signals were 3D-rendered using IMARIS (Bitplane, Switzerland). To analyze the intracytoplasmic localization of NG2 within activated microglia and macrophages, NG2 signals outside of these cells were subtracted to reveal only the co-localized signals using mask properties.

To simultaneously detect apoptotic cells in conjunction with NG2 expression, we performed double-labeling for terminal deoxynucleotidyl transferase dUTP nick end labeling (TUNEL) according to the manufacturer's protocol (Roche Diagnostics Corporation, Indianapolis, IN, USA) and NeuN using mouse monoclonal antibodies (1:400; Millipore). This was followed by $1 \mathrm{~h}$ incubation with Cy3-conjugated streptavidin (1:1200; Jackson ImmunoResearch) for the TUNEL method and Alexa Fluor 488-tagged goat anti-mouse antibody (1:300; Thermo Fisher). Counterstaining of cell nuclei was carried out with DAPI for 10 $\min$.

\section{Quantitative analysis}

To quantify time-dependent changes in the number of NG2 glia in the pyramidal cell layer and the stratum radiatum of the CA1 hippocampus, three coronal sections double-labeled for NG2 and Ibal from control and ischemic rats at days $3,7,14$, and $28(\mathrm{n}=3$ per time point) were obtained from the invariable region $-2.8 \mathrm{~mm}$ to $-3.8 \mathrm{~mm}$ dorsal to bregma [37]. Only cells with their nuclei in the plane of the section were recorded. Two measurements were performed because of the differences in the characteristic cytoarchitectonic features of hippocampal layers, i.e., one in the smaller pyramidal cell layer with its densely packed stacks of neuronal somata and the other in the wide and relatively soma-free layer called the stratum radiatum. For CA1 pyramidal cell measurements, five areas from the middle third of the CA1 subfield were chosen along $160 \mu \mathrm{m}$ of the pyramidal band in each hemisphere and were captured at $400 \times$ magnification under constant viewing conditions. The labeled cells of the stratum radiatum were counted in five areas $(160 \times 160 \mu \mathrm{m}$ per field $)$ in each hemisphere. The results of these cell counts are presented as the mean \pm standard error of the mean (SEM). Data analysis was performed using one-way analysis of variance (ANOVA) followed by Bonferronis multiple comparisons test. Differences with $\mathrm{p}$ values less than 0.05 were considered statistically significant. All statistical analyses were performed using GraphPad Prism version 5 (GraphPad Software Inc.; San Diego, CA). To quantify the numbers of proliferating NG2 glia, sections triple-labeled for NG2, Iba1, and Ki67 from control and ischemic rats at days $3,7,14$, and 28 ( $\mathrm{n}=3$ per time point) were obtained, as described above. The percentage of proliferating NG2 glia are expressed as the number of $\mathrm{NG}^{+} / \mathrm{Ki} 67^{+} / \mathrm{Ibal}^{-}$cells out of 
the total number of $\mathrm{NG2}^{+} / \mathrm{Ibal}^{-}$cells. The results are presented as the mean \pm SEM. Data analysis was performed as described above.

We quantified time-dependent changes in intensity profiles of NG2 expression in the pyramidal cell layer and the stratum radiatum of the CA1 hippocampus. Three sections taken from control and ischemic rats at days $3,7,14$, and 28 ( $\mathrm{n}=3$ per time point) were obtained from the invariable region $-2.8 \mathrm{~mm}$ to $-3.8 \mathrm{~mm}$ dorsal to bregma [37]. Five randomly selected areas of $50 \times 50 \mu \mathrm{m}$ were chosen in the pyramidal cell layer and in the stratum radiatum of each section, and all images were captured at $400 \times$ magnification under constant viewing conditions. The confocal images were analyzed using ZEN Blue 2.3 (Carl Zeiss AG, Oberkochen, Germany). The data are expressed in arbitrary units as mean \pm SEM. Data analysis was performed using one-way ANOVA followed by Bonferronis multiple comparisons test. Differences with $\mathrm{p}$ values less than 0.05 were considered statistically significant.

Additionally, we assessed the relative intensity of NG2 immunoreactivity in activated microglia/macrophages in both the pyramidal cell layer and the stratum radiatum of the CA1 hippocampus. Three double-labeled sections for NG2 and Ibal were taken from control and ischemic rats at days $3,7,14$, and 28 ( $\mathrm{n}=3$ per time point) as described above. Five randomly selected areas of $50 \times 50$ $\mu \mathrm{m}$ were chosen in the pyramidal cell layer and in the stratum radiatum of each section, and all images were captured at 400× magnification under constant viewing conditions. The confocal images were analyzed using ZEN Blue 2.3 (Carl Zeiss AG). The data are expressed in arbitrary units as mean \pm SEM. Data analysis was performed using two-way ANOVA followed by Bonferronis multiple comparisons test. Differences with p values less than 0.05 were considered statistically significant.

\section{Immunoelectron microscopic study}

For correlative light and electron microscopic study, vibratome sections (100 $\mu \mathrm{m}$ thick) containing the CAl region of the hippocampus from control $(n=3)$ and ischemic $(n=3)$ rats at days 3 and 14 were cryoprotected with $2.3 \mathrm{M}$ sucrose in $0.1 \mathrm{M}$ phosphate buffer and frozen in liquid nitrogen. Semi-thin cryosections $(2 \mu \mathrm{m}$ thick) were cut at $-100^{\circ} \mathrm{C}$ with a glass knife in a Leica EM UC7 ultramicrotome equipped with the FC7 cryochamber (Leica, Wetzlar, Germany). The sections were double-labeled using a mix of rabbit polyclonal antibody against NG2 (1:500; Millipore) and goat polyclonal antibody against Iba1 (1:500; Abcam). Antibody staining was visualized using Cy3-conjugated donkey anti-goat antibody (1:2000; Jackson ImmunoResearch) and Alexa Fluor 488-tagged goat anti-rabbit antibody (1:300; Thermo Fisher). Sections were additionally labeled with DAPI for $10 \mathrm{~min}$. Coverslipped sections were examined with a confocal microscope and photographed at various magnifications with a differential interference contrast setting to find specific areas for a later examination by electron microscopy. After the coverslips had been floated off the sections, the tissues were prepared further for electron microscopy. After postfixation, dehydration, and embedding in Epon 812 (Polysciences, Warrington, PA, USA), areas of interest were excised and glued onto resin blocks. After being cut into ultrathin sections of 70 90 $\mathrm{nm}$ thickness, they were observed using an electron microscope (JEM 1010; JEOL, Tokyo, Japan) with slight uranyl acetate staining. For pre-embedding immunoelectron microscopy, floating vibratome sections $(50 \mu \mathrm{m}$ thick) from ischemic rats $(\mathrm{n}=3)$ at day 14 were immunostained with rabbit polyclonal antibody against NG2 (1:500; Millipore). Immunoreactions were visualized using peroxidase-labeled goat anti-rabbit IgG (1:200; Jackson ImmunoResearch) and 0.05\% 3,3'-diaminobenzidine tetrahydrochloride (DAB) as a chromogen. The tissues were prepared further for electron microscopy, as described above.

\section{RESULTS}

Spatiotemporal profiles of NG2-positive cells in relation to neuronal damage in the hippocampal CA1 region after transient forebrain ischemia

We first examined neurodegeneration in the CA1 hippocampus after transient forebrain ischemia using immunostaining for TUNEL and the neuron-specific marker NeuN. No specific TUNEL staining was detected in the pyramidal cell layers of control hippocampi, in which the pyramidal cells were densely distributed (Fig. 1A). Three days after reperfusion, NeuN immunoreactivity in the pyramidal cell layer declined, and most pyramidal neurons were positive for TUNEL, a marker of cell death (Fig. 1B and 1C), as described previously $[35,38,39]$. Experimental rats had reproducible cell death in the CA1 hippocampus after ischemia. In addition, we performed immunohistochemical analysis for MAP2, which is known to be an early indicator of neurodegeneration [40]. In control animals, MAP2 immunoreactivity was clearly observed in dendrites in the stratum radiatum as well as in the somata of CA1 neurons (Fig. 1D). Three days after reperfusion, reduced staining for MAP2 in the somata and dendrites of CA1 neurons was evident when compared to controls (Fig. 1E), and a profound loss of MAP2 immunoreactivity was observed in CA1 neurons at 14 days (Fig. 1F).

Sequential temporal profiles of neuronal and neuroglial alterations in the acute (within 3 days of injury), subacute (7 14 days post-injury), and chronic phases (>14 days post-injury) within vulnerable CA1 hippocampus have been well documented after transient forebrain ischemia $[33,41]$. Thus, we next defined the 


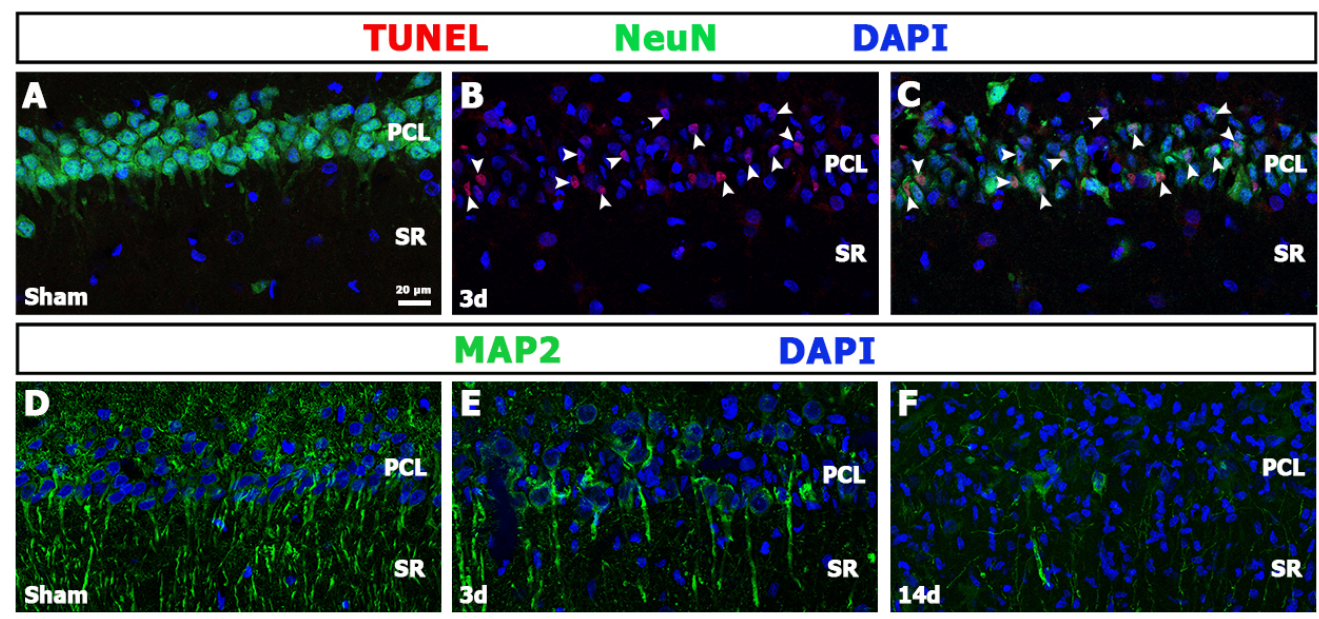

Fig. 1. Representative images showing neurodegeneration in the CA1 region of the hippocampus following transient forebrain ischemia. (A C) Double-labeling for the neuron-specific marker NeuN and the cell death marker terminal deoxynucleotidyl transferase dUTP nick end labeling (TUNEL) in the hippocampal CA1 region of sham-operated rats (A) and ischemic rats, 3 days after reperfusion (B and C). Note the lack of TUNEL-specific staining in the pyramidal cell layer of saline-treated controls, whereas most pyramidal neurons of the ischemic animals are positive for TUNEL (arrowheads). (D F) Changes in immunoreactivity for microtubule-associated protein 2 (MAP2) in the CA1 hippocampus after ischemia. (D) In sham-operated rats, extensive labeling for MAP2 is noted in both somata and dendrites in the CA1 hippocampus. (E) Three days after reperfusion, loss of both somatic and dendritic labeling for MAP2 is evident in the CA1 hippocampus. (F) At 14 days, only a few remaining dendrites are noted in the CA1 hippocampus. Cell nuclei are stained with 4,6-diamidino-2-phenylindole (DAPI). Scale bars represent $20 \mu \mathrm{m}$ in A F.

relationship between the distribution of NG2 expression and neurodegeneration in the ischemic CA1 hippocampus using doublelabeling for NG2 and NeuN during a 28-day post-ischemic period. In the CA1 hippocampus of controls, weak immunoreactivity for NG2 was detected in small stellate cells with fine processes localized throughout all strata (Fig. 2A and 2B). At 3 days after reperfusion, NG2 immunoreactivity appeared to increase in the CA1 hippocampus with prominent enrichment in the pyramidal cell layer (Fig. 2C and 2D). At 7 days after reperfusion when NeuN immunoreactivity had virtually disappeared in the pyramidal cell layer of the CA1 hippocampus, increased NG2 immunoreactivity was evident in the stratum radiatum in addition to that in the pyramidal cell layer (Fig. 2E and 2F), and there was a further increase in NG2 immunoreactivity in both strata of the CA1 region by day 14 (Fig. $2 \mathrm{G}$ and 2H). At 28 days after reperfusion, NG2 immunoreactivity appeared to decrease in the stratum radiatum, but it remained with prominent enrichment in the pyramidal cell layer (Fig. 2I and 2J). Thus, these data indicated the increased expression of NG2 in the ischemic CA1 hippocampus in comparison to sham-operated controls. We next compared the relative intensity of NG2 immunoreactivity within the pyramidal cell layer and the stratum radiatum in the CA1 hippocampus of sham-operated and ischemic rats using computerized image analysis. The statistical analysis revealed that the NG2 staining intensities in the pyramidal cell layer and the stratum radiatum shared a similar temporal pattern; the NG2 expression in both layers was significantly in- creased and peaked at 7 days, and this enhanced expression was maintained for up to 28 days (Fig. $2 \mathrm{~K}$ and $2 \mathrm{~L}$ ).

\section{Distribution and morphology of NG2 glia in the hippocam- pal CA1 region of sham-operated rats}

Several reports have provided evidence that NG2 expression is also induced in activated microglia/macrophages following an injury to the CNS [16-19, 21, 22, 24, 42]. Thus, we aimed at identifying the cellular phenotypes of NG2-positive cells using double-labeling for NG2 and the microglial marker Iba1. In shamoperated controls, Iba1-negative NG2 glia were identified in both the pyramidal cell layer and the stratum radiatum of the CA1 hippocampal region (Fig. 3A). Higher-magnification images (Fig. 3B and $3 \mathrm{C}$ ) and the three-dimensional (3D) reconstruction using 3D rendering in IMARIS (Fig. 3D) clearly revealed that these resting NG2 glia shared similar morphological features in both strata and that they were comprised of small stellate cells from which multiple fine processes radiated in all directions.

To define whether NG2 expression is detected in astrocytes in the hippocampal CA1 region of sham-operated rats, we performed double-labeling with NG2 and GFAP. As shown in Fig. 3E, no specific NG2 staining was detected in astrocytes, which displayed thin glial processes. Moreover, double-labeling with NG2 and APC, a marker of mature oligodendrocytes [43], revealed a nonoverlapping expression of NG2 and APC, despite APC-positive oligodendrocytes being clearly observed in both the pyramidal 

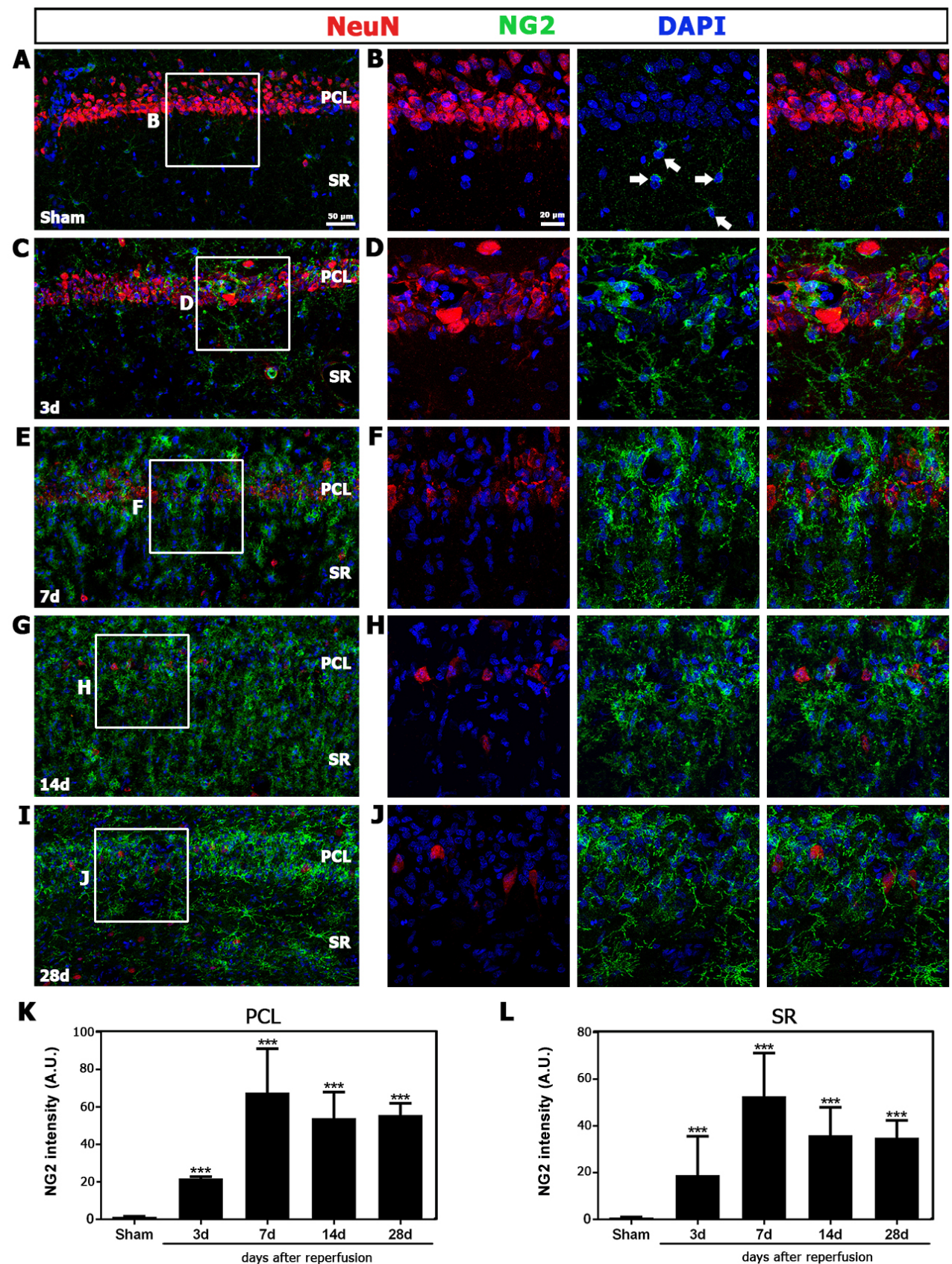

Fig. 2. Representative images showing the spatiotemporal profiles of NG2-positive cells in the CA1 region of the hippocampus following transient forebrain ischemia. (A J) Double-labeling for NeuN and NG2 was performed on free-floating sections. (A, B) Weak NG2 expression is observed in small stellate cells (arrows) in both the pyramidal cell layer (PCL) and the stratum radiatum (SR) in the hippocampal CA1 region of sham-operated rats. The boxed area of the pyramidal cell layer (PCL) and stratum radiatum (SR) in A are enlarged in B. Note that pyramidal neurons are densely distributed in the pyramidal cell layer. (C, D) At day 3 after reperfusion, NG2 immunoreactivity appears to be increased in the CA1 hippocampus with prominent enrichment in the pyramidal cell layer, in which NeuN expression declines. $(\mathrm{E} \sim \mathrm{H}) \mathrm{NG} 2$ immunoreactivity tends to increase in both the pyramidal cell layer and the stratum radiatum at days $7(\mathrm{E}, \mathrm{F})$ and $14(\mathrm{G}, \mathrm{H})$ after reperfusion. Note that NeuN immunoreactivity has disappeared in the pyramidal cell layer by day 7. (I, J) At day 28 after reperfusion, NG2 immunoreactivity is still prominent in the pyramidal cell layer but appears to decrease in the stratum radiatum. Cell nuclei are stained with DAPI. Scale bars represent $50 \mu \mathrm{m}$ in A, C, E, G and I, and $20 \mu \mathrm{m}$ in B, D, F, H, and J. (K, L) Quantitative analysis of the intensity of NG2 expression in the pyramidal cell layer (K) and the stratum radiatum (L) in sham-operated control and ischemic rats. Note that the NG2 staining intensities in both the pyramidal cell layer and stratum radiatum significantly increase and peak at 7 days after reperfusion, and this enhanced expression is maintained for up to 28 days. $\mathrm{n}=3$ rats per group. In each animal, five randomly selected areas in both strata of each section were analyzed in each of three sections. The data are expressed in arbitrary units (A.U.) as mean \pm SEM. ${ }^{* \star} \mathrm{p}<0.001$ vs sham-operated controls based on oneway ANOVA followed by Bonferroni's multiple comparisons test. 

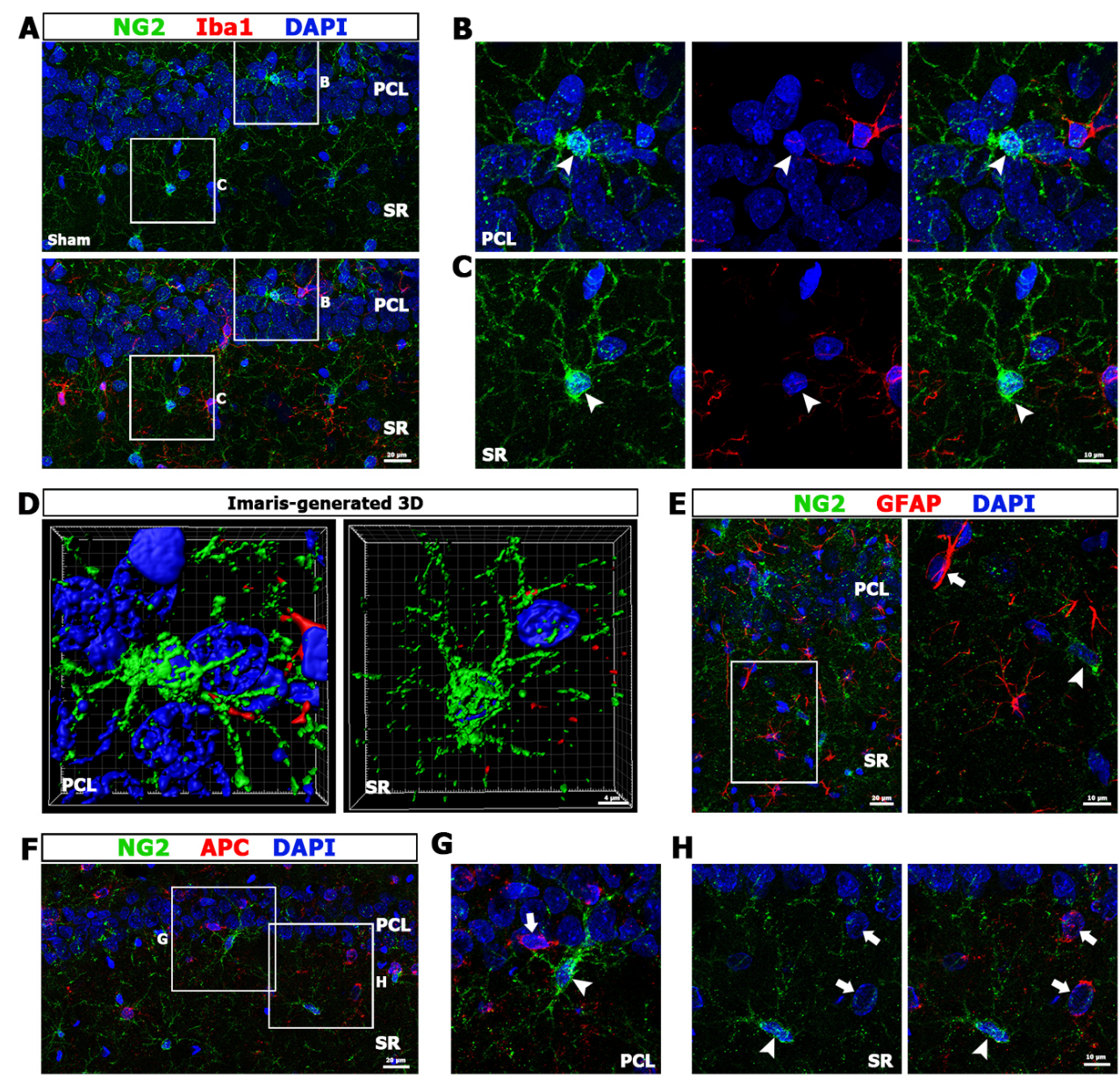

Fig. 3. Characterization of NG2 glia in the hippocampal CA1 region of sham-operated rats using double-labeling for NG2 and Iba1. (A C) Lower(A) and higher- (B, C) magnification views. The boxed areas of the pyramidal cell layer (PCL) and stratum radiatum (SR) in A are enlarged in B and C, respectively. NG2 glia and Ibal-positive microglia do not have overlapping distributions in the CA1 hippocampus. Note that resting NG2 glia in the pyramidal cell layer (arrowheads in B) and the stratum radiatum (arrowheads in C) have multiple fine processes radiating in all directions. (D) Threedimensional renderings of the images shown in B and C, showing that NG2 glia share similar morphological characteristics in both strata of the CA1 hippocampus. (E) Double-labeling for NG2 and GFAP in the CA1 hippocampus of sham-operated rats. The boxed area in the left panel is enlarged in the right panel. Note that NG2 glia (arrowhead) and astrocytes (arrow) do not have overlapping distributions in the CA1 hippocampus. (F H) Doublelabeling for NG2 and APC, a marker of mature oligodendrocytes, in the CA1 hippocampus of sham-operated rats. The boxed areas of the pyramidal cell layer and stratum radiatum in F are enlarged in G and H, respectively. Note that NG2 glia (arrowheads) are distinct from mature oligodendrocytes (arrows). Cell nuclei are stained with DAPI. Scale bars represent $20 \mu \mathrm{m}$ in A, left panel of E and F; $10 \mu \mathrm{m}$ in B, C, right panel of E, G and $\mathrm{H}$; and $4 \mu \mathrm{m}$ in D.

cell layer and the stratum radiatum of the CA1 hippocampus (Fig. $3 \mathrm{~F}$ and $3 \mathrm{H})$.

\section{Time-dependent changes in the distribution and morphol-} ogy of NG2 glia in the hippocampal CA1 region after transient forebrain ischemia

We next defined the spatial and temporal features of NG2 glia in the ischemic CA1 hippocampus using double-labeling for NG2 and Ibal. In the CA1 hippocampus reperfused for 3 days, prominent NG2 expression appeared in both the pyramidal cell layer and the stratum radiatum in comparison to that observed in the control hippocampus, whereas prominent Ibal immunoreactivity was detected only in the pyramidal cell layer (Fig. 4A). At higher magnifications (Fig. 4B and 4C), $\mathrm{NG2}^{+} / \mathrm{Ibal}^{-}$cells, namely NG2 glia exhibited morphologies similar to typical reactive NG2 glia with larger cell bodies and thick processes, as reported previously $[16,44,45]$, but they appeared to differ in their entire cellular morphology and arborization in the two strata of the CA1 hippocampus. When comparing NG2 glia in the stratum radiatum and in the pyramidal cell layer, the former exhibited an increase in the number and complexity of branched processes, whereas the latter had fewer and thicker processes (Fig. 4B and 4C). At this time point, double-labeling with NG2 and the two glia-specific markers GFAP or APC revealed that reactive NG2 glia were devoid of spe- 

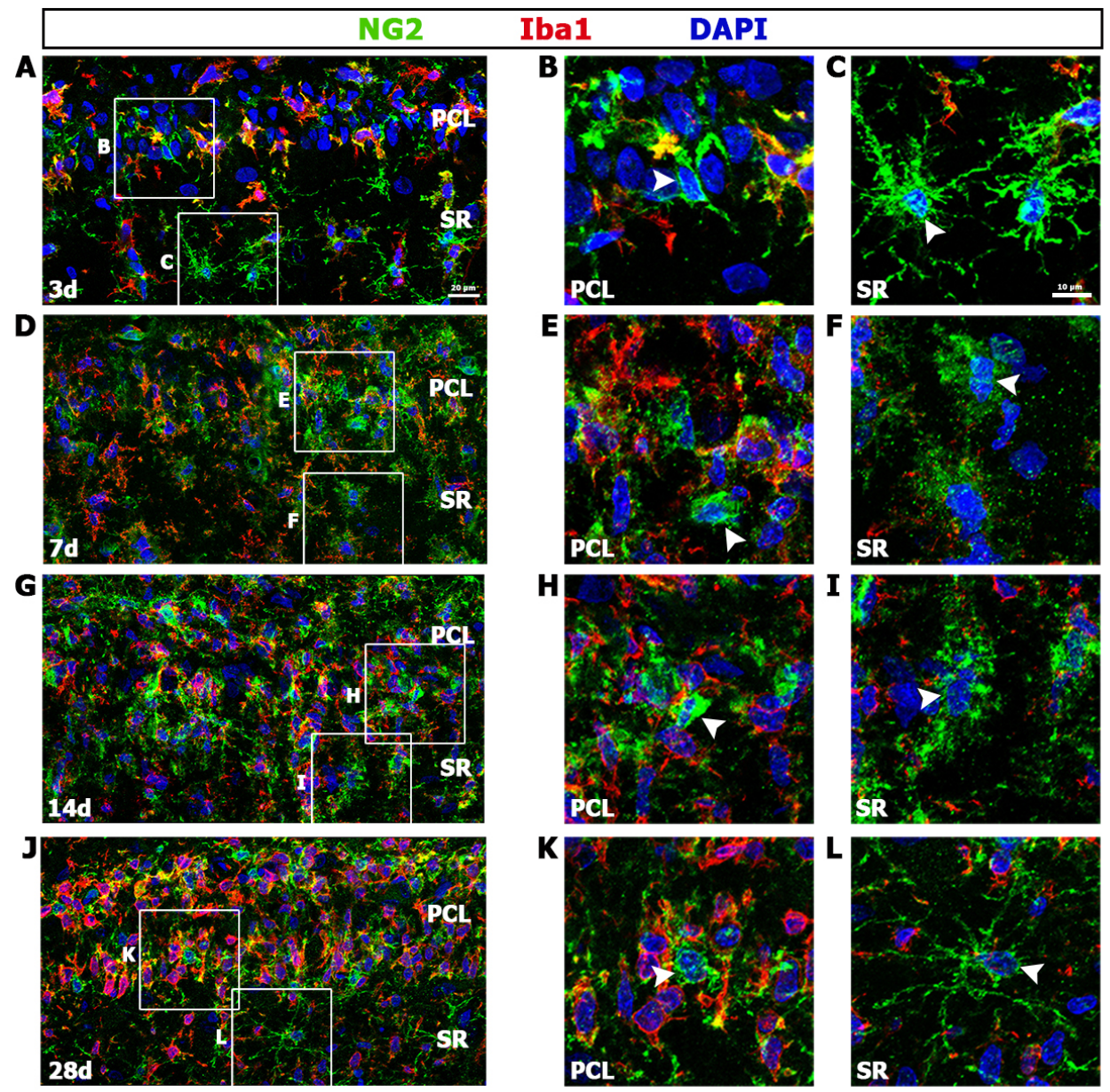

Fig. 4. Characterization of NG2 glia in the hippocampal CA1 region of rats at days 3, 7, 14, and 28 following transient forebrain ischemia using doublelabeling for NG2 and Iba1. (A C) Lower- (A) and higher- (B, C) magnification views of the CA1 hippocampus 3 days after reperfusion. The boxed areas of the pyramidal cell layer (PCL) and stratum radiatum (SR) are enlarged in B and C, respectively. The numbers of NG2 glia (NG2 $/ \mathrm{Iba1}^{-}$cells) appear to increase in both the pyramidal cell layer and the stratum radiatum, whereas NG2-positive microglia/macrophages are preferentially localized to the pyramidal cell layer. Notably, NG2 glia in the pyramidal cell layer (arrowhead in B) have fewer and thicker processes than those in the stratum radiatum (arrowhead in C). (D F) Lower- (D) and higher- (E, F) magnification views of the CA1 hippocampus 7 days after reperfusion showing that prominent NG2 and Ibal expression appears in both the pyramidal cell layer and the stratum radiatum. Note that NG2 glia in the stratum radiatum (arrowhead in F) exhibit more numerous and complex branching processes compared to those in the pyramidal cell layer (arrowhead in E). (G I) Lower- (G) and higher- (H, I) magnification views of the CA1 hippocampus 14 days after reperfusion. Immunoreactivities for NG2 and Ibal are still prominent in both the pyramidal cell layer and the stratum radiatum. Note that NG2 glia in the pyramidal cell layer (arrowhead in $\mathrm{H}$ ) have fewer and thicker processes compared to those in the stratum radiatum (arrowhead in I). (J L) Lower- $(\mathrm{J})$ and higher- $(\mathrm{K}, \mathrm{L})$ magnification views of the CA1 hippocampus 28 days after reperfusion, showing that immunoreactivity for NG2 is still observed in the pyramidal cell layer and the stratum radiatum, whereas Ibal appears to be preferentially localized to the pyramidal cell layer. Note that NG2 glia in the pyramidal cell layer (arrowhead in K) have thick and short processes, while NG2 glia in the stratum radiatum (arrowhead in L) have numerous complex branching processes. Cell nuclei are stained with DAPI. Scale bars represent: $20 \mu \mathrm{m}$ in A, D, G and J; $10 \mu \mathrm{m}$ in B, C, E, F, H, I, K and L.

cific labeling for both GFAP and APC (Fig. 5). At day 7 after reperfusion, increased expression levels for Ibal and NG2 were noted in both strata of the CA1 hippocampus compared with those at day 3 (Fig. 4D). Higher-magnification images revealed that NG2 glia in the stratum radiatum had more multiple and complex branching processes compared with those in the pyramidal cell layer, which had large cell bodies with few processes (Fig. $4 \mathrm{E}$ and $4 \mathrm{~F}$ ).

Fourteen days after reperfusion, the labeling patterns for NG2 and Iba1 in the CA1 hippocampus were similar to those observed at day 7 , although there was a further increase in NG2 expression in the stratum radiatum (Fig. 4G). As shown in highermagnification views (Fig. 4H and 4I) NG2 glia presented at day 14 morphological characteristics similar to those at day 7. Particularly, the shape and arborization of single processes in reactive NG2 glia were not almost discernible in the stratum radiatum because amorphous clouds of NG2 staining were localized around 

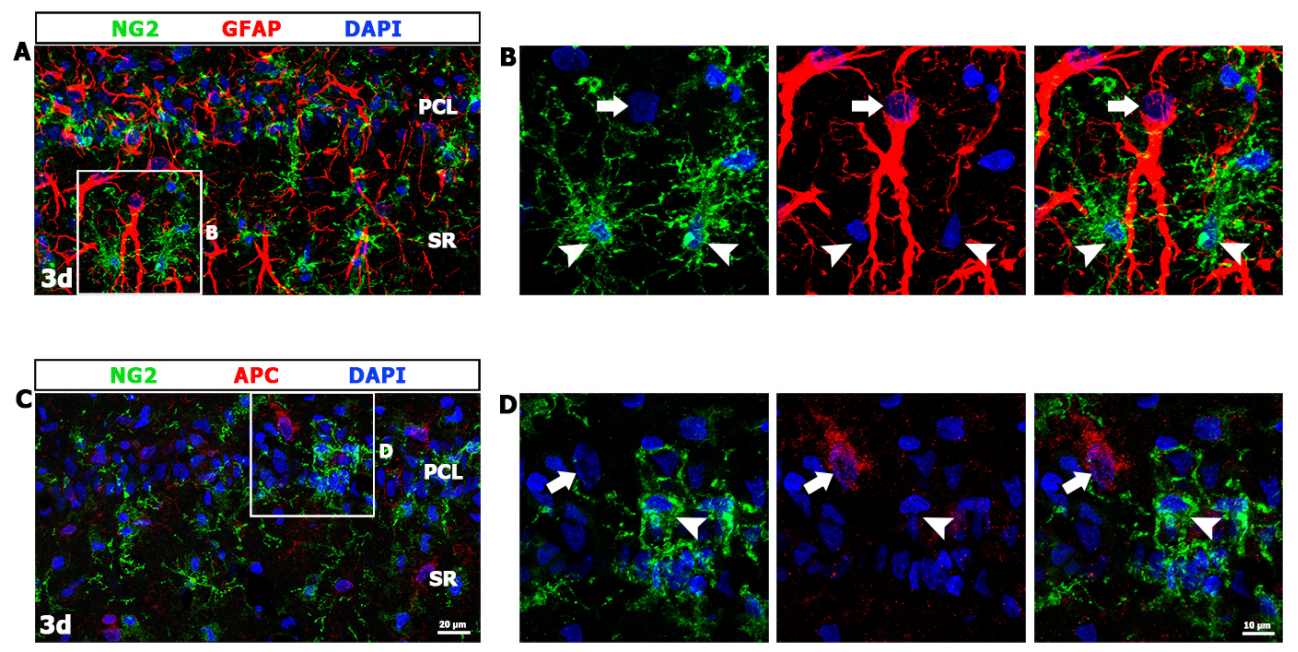

Fig. 5. Representative images indicating the non-overlapping expression of NG2 glia and either astrocytes or mature oligodendrocytes in the hippocampal CA1 region of rats at day 3 after transient forebrain ischemia. (A, B) Double-labeling for NG2 and GFAP in the pyramidal cell layer (PCL) and stratum radiatum (SR) of the ischemic CA1 hippocampus showing that reactive NG2 glia (arrowheads) and reactive astrocytes (arrows) have nonoverlapping distributions. The boxed area in A is enlarged in B. (C, D) Double-labeling for NG2 and APC in the ischemic CA1 hippocampus showing that NG2 glia (arrowheads) are distinct from mature oligodendrocytes (arrows). The boxed area in c is enlarged in D. Cell nuclei are stained with DAPI. Scale bars represent $20 \mu \mathrm{m}$ in $\mathrm{A}$ and $\mathrm{C}, 10 \mu \mathrm{m}$ in B and D.

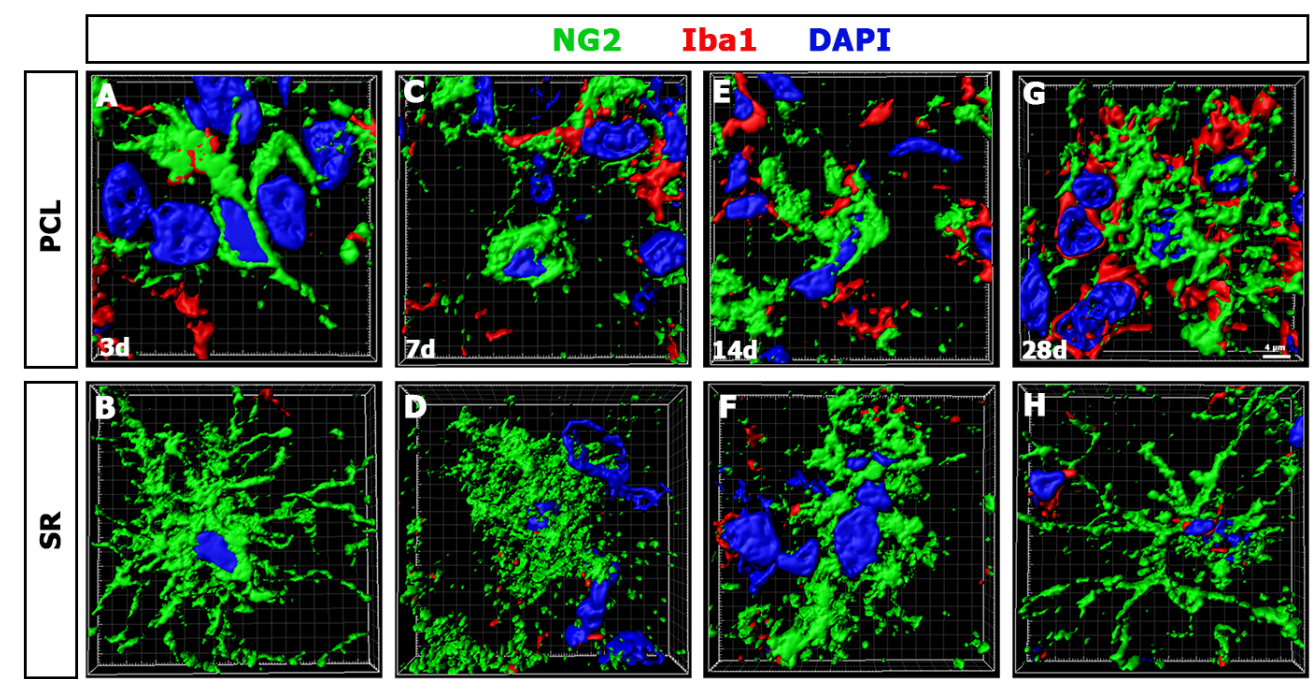

Fig. 6. The morphological evolution of NG2 glia in the hippocampal CA1 region of rats 3, 7, 14, and 28 days following transient forebrain ischemia. Three-dimensional renderings of NG2 glia are shown in Figures 4B, C, E, F, H, I, K, and L, respectively. Note that NG2 glia exhibit obvious differences in their cellular morphology and arborization of the processes between the two layers. Additionally, note that NG2 glia in the stratum radiatum at $7 \sim 14$ days after reperfusion are surrounded by amorphous clouds of NG2 staining and, thus, their individual processes cannot be discerned. Cell nuclei are stained with DAPI. Scale bars represent $4 \mu \mathrm{m}$ in A H.

the NG2 glia. On day 28 after reperfusion, the latest time point examined, NG2 expression was still observed in both strata of the CA1 hippocampus, but NG2-positive activated microglia/macrophages appeared to be preferentially localized in the pyramidal cell layer (Fig. 4J). Using higher-magnification views (Fig. $4 \mathrm{~K}$ and $4 \mathrm{~L}$ ), we could demonstrate that NG2 glia in the stratum radiatum converted into the typical reactive phenotypes with multiple and highly branched processes being clearly identified, while those in the pyramidal cell layer had fewer and shorter processes.

We were able to demonstrate, using enhanced visualization through 3D rendering approaches, that the morphologies of NG2 glia were altered in the two strata of the CA1 hippocampus dur- 


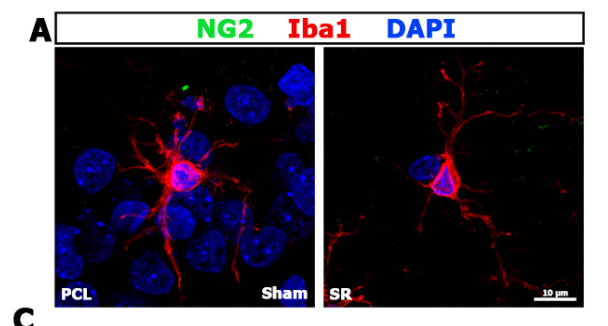

C
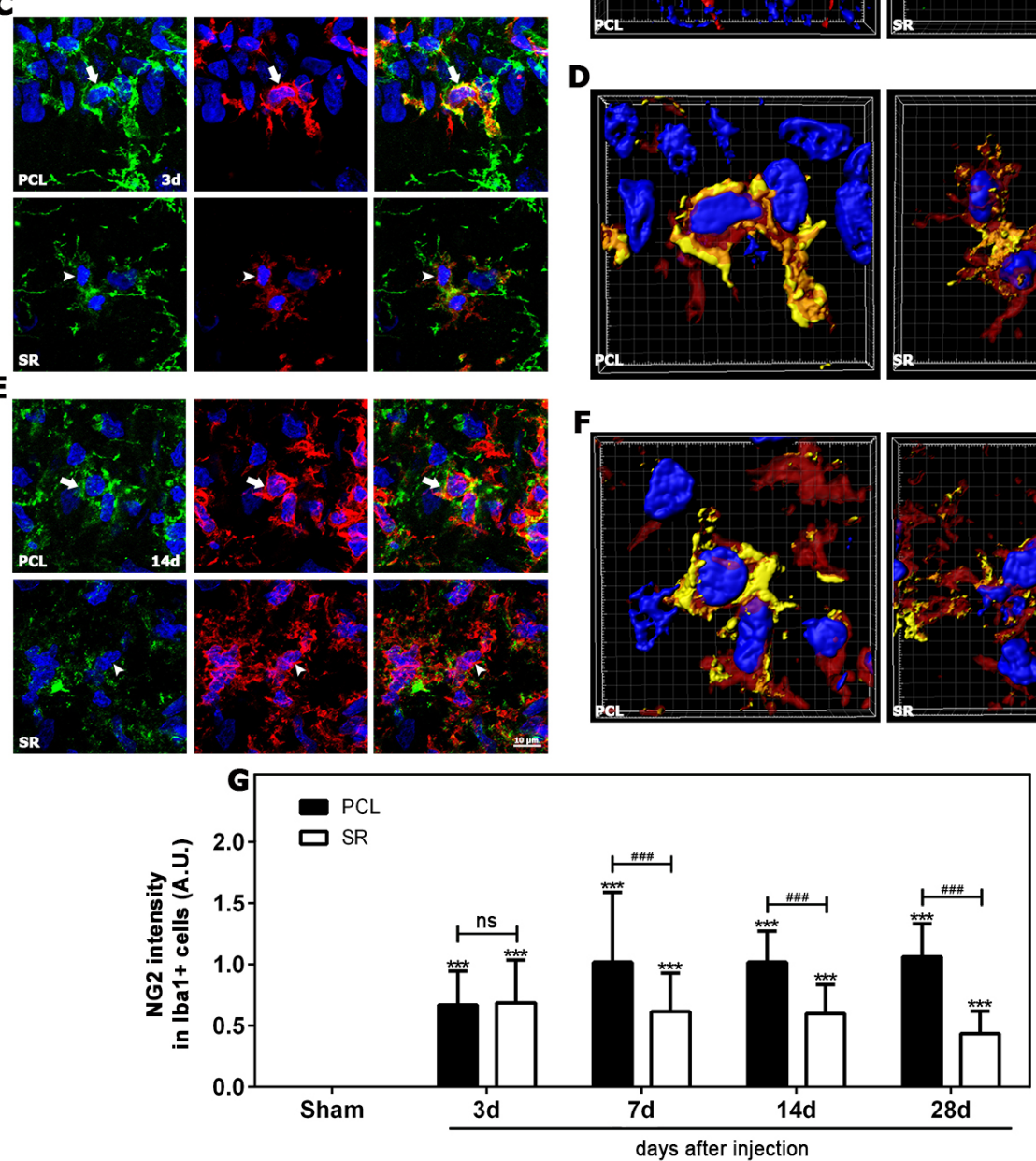

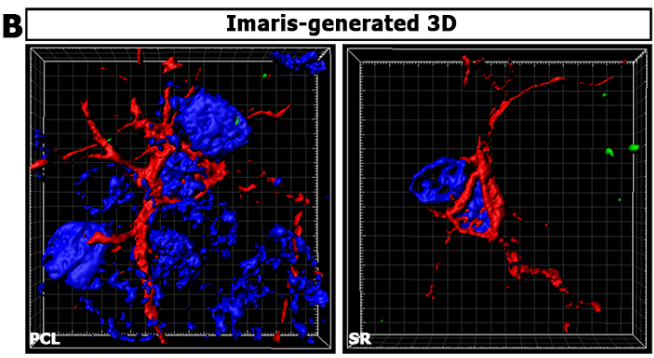

D
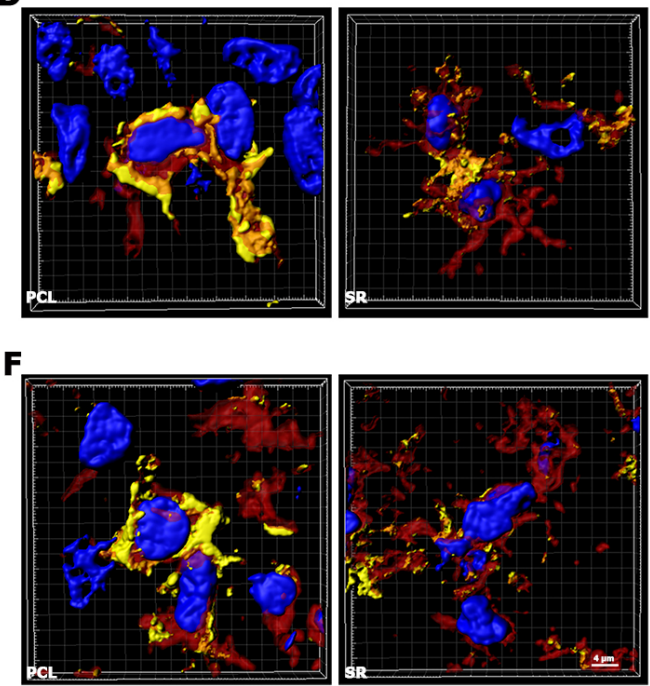

Fig. 7. Characterization of NG2-positive microglia/macrophages in the CA1 region of the hippocampus following transient forebrain ischemia using double-labeling for NG2 and Iba1. (A) Resting microglia in both the pyramidal cell layer (PCL) and the stratum radiatum (SR) are devoid of NG2 in the hippocampal CA1 region of sham-operated rats. (B) The three-dimensional renderings of the above images indicate that the resting microglial cells of both strata exhibit a ramified morphology. (C) At 3 days after reperfusion, NG2 expression is induced in amoeboid-like macrophages with retracted processes (arrows) in the pyramidal cell layer, as well as in activated stellate microglial cells with evident processes (arrowheads) in the stratum radiatum. (D) The three-dimensional renderings of the above images indicate that NG2 expression is more prominent in activated microglia/macrophages of the pyramidal cell layer compared those of the stratum radiatum. (E) At 14 days after reperfusion, the labeling patterns of NG2 expression in activated microglia/macrophages in the pyramidal cell layer (arrows) and the stratum radiatum (arrowheads) remain unchanged in comparison to the 3-day time point. (F) Three-dimensional renderings of the above images, indicating that NG2 expression is more prominent in amoeboid-like macrophages in the pyramidal cell layer than in activated stellate microglia in the stratum radiatum. Cell nuclei are stained with DAPI. (G) Quantitative temporal analysis of the intensity of NG2 immunoreactivity in activated microglia/macrophages in the CA1 hippocampus during the post-ischemic period. Note that the relative intensity of microglial NG2 immunoreactivity in the pyramidal cell layer was significantly higher than that in the stratum radiatum by 7 days after reperfusion. $\mathrm{n}=3$ rats per group. In each animal, five randomly selected areas in both strata of each section were analyzed in each of three sections. The data are expressed in arbitrary units (A.U.) as mean \pm SEM. ns, not significant; ${ }^{* * *} \mathrm{p}<0.001$ vs sham-operated controls; ${ }^{* * *} \mathrm{p}<0.001 \mathrm{vs}$ pyramidal cell layer based on two-way ANOVA followed by Bonferroni's multiple comparisons test. Scale bars represent $10 \mu \mathrm{m}$ in A, C, and E; and $4 \mu \mathrm{m}$ in B, D, and F. 
ing the 28 days following ischemia (Fig. 6). In particular, the 3D renderings revealed that NG2 glia in the stratum radiatum at 7 14 days after reperfusion were almost surrounded by amorphous clouds of NG2 staining, which did not allow the clear identification of their individual processes (Fig. 6).

\section{Induction of NG2 expression in activated microglia/mac-} rophages in the hippocampal CA1 region of ischemic rats

We next examined the induction of NG2 expression in activated microglia/macrophages in the ischemic CA1 hippocampus. In sham-operated rats, no significant immunoreactivity for NG2 was detected in resting microglia with ramified morphology, and there was no apparent difference in shape between the pyramidal cell layer and the stratum radiatum (Fig. $7 \mathrm{~A}$ and $7 \mathrm{~B}$ ). In the CA1 hippocampus reperfused for 3 days, evident NG2 expression was noted in Iba1-positive microglia located in the pyramidal cell layer and the stratum radiatum, where they displayed the characteristic features of amoeboid-like macrophages with retracted processes and those of activated stellate microglial cells with thick and short processes, respectively (Fig. 7C). In particular, the 3Drendered images clearly demonstrated that NG2 expression was observed on the surface or within the cytoplasm of these microglia/macrophages, and the expression was more prominent in brain macrophages in the pyramidal cell layer (Fig. 7D). At 14 days after reperfusion, the labeling patterns of microglial NG2 expression in both strata of the CA1 hippocampus remained unchanged in comparison to those at day 3 (Fig. 7E and 7F). This observation was confirmed by quantitative temporal analysis showing that the mean fluorescence intensity of microglial NG2 expression in both the pyramidal cell layer and the stratum radiatum was significantly increased throughout the injury period (Fig. 7G). In addition, the relative intensity of microglial NG2 immunoreactivity in the pyramidal cell layer was significantly higher than that in the stratum radiatum throughout the injury period, although its intensity in both strata was similar at 3 days after reperfusion.

\section{Quantitative analysis of time- and lamina-dependent dis- tribution of NG2 glia in the hippocampal CA1 region of sham-operated and ischemic rats}

As described above, the numbers of NG2-positive cells appeared to be increased in the CAl hippocampus after transient forebrain ischemia. Thus, we quantified the numbers of NG2 glia in both examined strata of the CA1 hippocampus in control and ischemic rats. As shown in Fig. $8 \mathrm{~A}$ and 8B, the numbers of NG2 glia in the stratum radiatum and the pyramidal cell layer were significantly higher in ischemic rats compared to sham-operated controls over the 28-day period after reperfusion. The number of NG2 glia in the pyramidal cell layer was significantly increased at day 3 after reperfusion and remained elevated until day 28 (Fig. 8A), while their numbers in the stratum radiatum increased progressively for 14 days after reperfusion, although the number of NG2 glia was slightly decreased on day 28 (Fig. 8B).

We next determined whether the increase in the numbers of NG2-positive cells in the ischemic CA1 hippocampus was due to new cells generated via proliferation. Triple-labeling for NG2, Iba1, and Ki67, a marker expressed in cells at all cell cycle phases except G0 [46, 47], revealed that NG2 glia and NG2-positive microglia frequently exhibited Ki67-positive nuclei in both the pyramidal cell layer (Fig. 8C) and the stratum radiatum (Fig. 8D). Thus, we quantified the numbers of NG2 glia proliferating in the two examined strata of the CAl hippocampus in control and ischemic rats. In the sham-operated CA1 hippocampus, the number of Ki67positive NG2 glia was negligible in the pyramidal cell layer and the stratum radiatum, but $24.4 \%$ and $29.6 \%$ of all NG2 glia in the pyramidal cell layer and the stratum radiatum, respectively, were proliferating at 3 days after reperfusion (Fig. $8 \mathrm{E}$ and $8 \mathrm{~F}$ ). This proportion declined in both layers thereafter, but it was maintained in the stratum radiatum at about $5 \%$ on day 28 , whereas only $1.3 \%$ of all NG2 glia were proliferating in the pyramidal cell layer at this time point.

\section{Ultrastructure of NG2 glia in the hippocampal CA1 region of sham-operated and ischemic rats}

Using a correlative light and electron microscopic approach, we then determined more precisely whether the ultrastructural morphology of NG2 glia was also different depending on the sub-layers of the CA1 hippocampus. Semi-thin sections double-labeled for NG2 and Iba1 were first observed using confocal microscopy, which clearly revealed NG2 glia devoid of Ibal immunoreactivity in both strata of the CA1 hippocampus of control rats (Fig. 9A and 9C). Overlay of the confocal and the transmission electron microscopic images demonstrated that resting NG2 glia had nuclei with clustered chromatin and a relatively narrow rim of cytoplasm with only a few stacks of rough endoplasmic reticulum. There was no obvious difference in their ultrastructural morphology between the two strata (Fig.9B and 9D).

In the CA1 hippocampus reperfused for 3 days, semi-thin sections double-labeled for NG2 and Ibal clearly revealed two types of NG2-positive cells, NG2 glia and activated microglia expressing NG2 in the pyramidal cell layer (Fig. 9E) and the stratum radiatum (Fig. 9G). When these cells were further analyzed with electron microscopy (Fig. 9F and 9H), we found that NG2 glia in the two strata shared ultrastructural features typical of reactive NG2 glia, and they had abundant rough endoplasmic reticulum and well- 

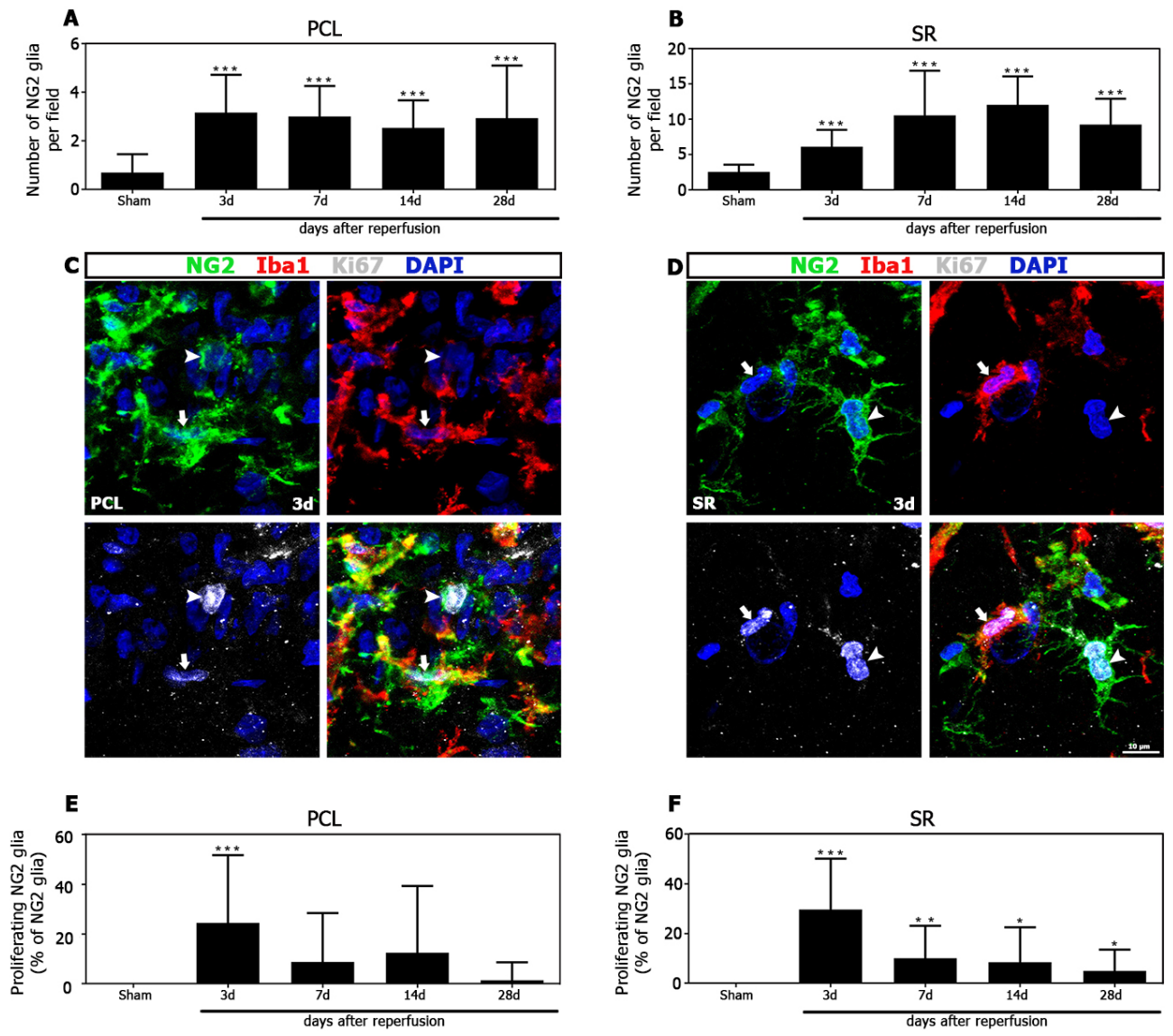

Fig. 8. Quantitative analysis of the number and the proliferation status of NG2 glia in the CA1 region of the hippocampus following transient forebrain ischemia. (A, B) The number of NG2 glia (namely, $\mathrm{NG2}^{+} / \mathrm{Iba1}^{-}$cells) in the pyramidal cell layer (PCL) was counted in five areas from the middle third of the CA1 subfield along $160 \mu \mathrm{m}$ of the pyramidal band in each hemisphere. The number of labeled cells in the stratum radiatum (SR) was counted in five areas $(160 \times 160 \mu \mathrm{m}$ per field) in each hemisphere. Three sections from three animals per group were included in this analysis. The numbers of NG2 glia in the pyramidal cell layer significantly increase, reach a maximum 3 days after reperfusion, and remain elevated until day 28 , while those in the stratum radiatum progressively increase until day 14 and then slightly decrease on day 28. (C, D) Representative images of triple-labeling for NG2, Iba1, and Ki67, showing that NG2 glia (arrowheads in C and D) and NG2-positive microglia/macrophages (arrows in C and D) have Ki67-labeled nuclei in both the pyramidal cell layer (C) and the stratum radiatum (D) at 3 days after reperfusion. Cell nuclei are stained with DAPI. (E, F) Temporal profile of proliferating NG2 glia in the pyramidal cell layer (E) and the stratum radiatum (F) in the hippocampal CA1 region after reperfusion. The number of NG2 ${ }^{+} /$ $\mathrm{Ibal}^{-} / \mathrm{Ki}^{+} 7^{+}$cells is divided by the total number of $\mathrm{NG}^{+} / \mathrm{Ibal}^{-}$cells for each area described above to indicate the percentage of proliferating NG2 glia. The proportion of Ki67-labeled NG2 glia is more than 20\% of all NG2 glia in both layers on day 3 and decreases abruptly on day 7. Note that in the stratum radiatum, this proportion remains at about $5 \%$ up to day 28 . The data are expressed as the mean $\pm \mathrm{SEM} .{ }^{*} \mathrm{p}<0.05,{ }^{* *} \mathrm{p}<0.01$, and ${ }^{* * *} \mathrm{p}<0.001$ vs shamoperated controls based on one-way ANOVA followed by Bonferronis multiple comparisons test. Scale bars represent $10 \mu \mathrm{m}$ in C and D.

developed Golgi complexes compared to resting NG2 glia.

Furthermore, we analyzed reactive NG2 glia in the CA1 hippocampus reperfused for 14 days. As shown in semi-thin sections double-labeled for NG2 and Iba1 (Fig. 10A and 10C), NG2 glia that were devoid of Ibal labeling were clearly distinguishable from NG2-positive microglia in both the pyramidal cell layer and the stratum radiatum. When the same semi-thin sections were further examined using transmission electron microscopy, we observed that reactive NG2 glia in both strata had a well-developed rough endoplasmic reticulum (Fig. 10B and 10D). We then defined the precise subcellular localization of NG2 within NG2 glia in the stratum radiatum using pre-embedding immunoelectron microscopy. As shown in Fig. 10E and 10F, NG2 immunoreactivity, as indicated by highly electron-dense $\mathrm{DAB}$ grains, was localized along the plasma membrane of NG2 glia and could be observed in the adjacent extracellular matrix.

\section{DISCUSSION}

We recently reported that NG2 glia undergo proliferation and morphological changes in the striatum of rats exposed to the mitochondrial toxin 3-nitropropionic acid [16]. In the present study, we 

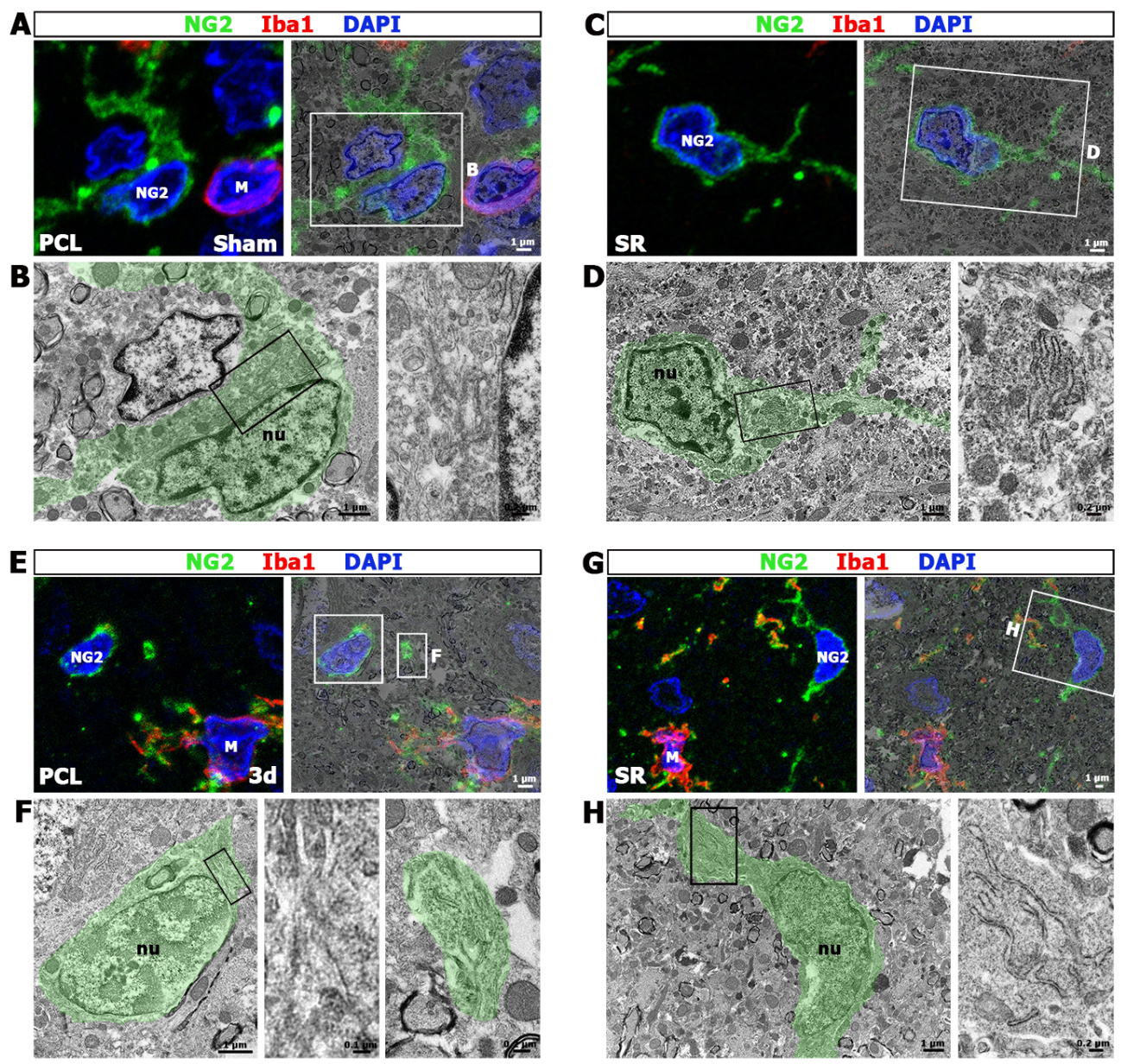

Fig. 9. Representative correlative light and electron microscopic images showing the ultrastructural characterization of resting and reactive NG2 glia in the hippocampal CA1 region. (A D) Ultrastructure of NG2 glia in the pyramidal cell layer (PCL; A, B) and stratum radiatum (SR; C, D) in the CA1 hippocampus of sham-operated rats. Confocal microscopic images of semi-thin sections double-labeled for NG2 and Ibal (left panels in A and C), images of confocal microscopic data overlaid onto the corresponding electron microscopic images (right panels in A and C), and the corresponding transmission electron microscopic images obtained from the same fields (left panels in B and D). The boxed areas in the left panels in B and D are enlarged in the right panels in B and D, respectively. Note that resting NG2 glia (shaded in green) in both layers have nuclei (nu) containing clustered chromatin, as well as scanty cytoplasm with few cytoplasmic organelles. (E H) Ultrastructure of NG2 glia in the pyramidal cell layer (E, F) and the stratum radiatum (G, $\mathrm{H}$ ) of ischemic rats at 3 days after reperfusion. Confocal microscopic images of semi-thin sections double-labeled for NG2 and Ibal (left panels in E and $\mathrm{G}$ ), images of confocal microscopic data overlaid onto the corresponding electron microscopic images (right panels in E and G), and the corresponding transmission electron microscopic images obtained from the same field (left panels in F and $\mathrm{H}$, right panel in F). The boxed areas in the left panels in F and $\mathrm{H}$ are enlarged in the middle panel in $\mathrm{F}$ and the right panel in $\mathrm{H}$, respectively. Notably, reactive NG2 glia (shaded in green) in the two examined layers have abundant rough endoplasmic reticulum and well-developed Golgi complexes. Cell nuclei are stained with DAPI. Scale bars represent $1 \mu \mathrm{m}$ in A, C, E, G and the left panels in B, D, F, and $\mathrm{H} ; 0.2 \mu \mathrm{m}$ in the right panels in $\mathrm{B}, \mathrm{D}$, and $\mathrm{H}$; and $0.1 \mu \mathrm{m}$ in the middle and right panels in $\mathrm{F}$.

demonstrated that NG2 glia can also transform into their reactive form in the ischemic CA1 hippocampus, where activation of NG2 glia showed a distinct temporal pattern in the pyramidal cell layer (somatic region) and the neighboring dendritic stratum radiatum. In addition, our data revealed that NG2 expression was induced in activated microglia/macrophages in the ischemic CA1 hippocampus, in a layer-specific manner. This is in agreement with our previous study showing the NG2 induction in activated microglia/ macrophages in the lesioned striatum [16]. Thus, supporting and extending our previous observations, the present study provides a detailed spatiotemporal characterization of reactive NG2 glia in the selectively vulnerable CA1 region of the rat hippocampus subjected to transient forebrain ischemia.

It is widely accepted that NG2 is a valuable marker for several types of cells including oligodendrocyte progenitors, pericytes, smooth muscle cells, and activated microglia/macrophages, in addition to NG2 glia (reviewed in $[4,5,48]$ ). The present data revealed that in the CA1 hippocampus of sham-operated controls, 

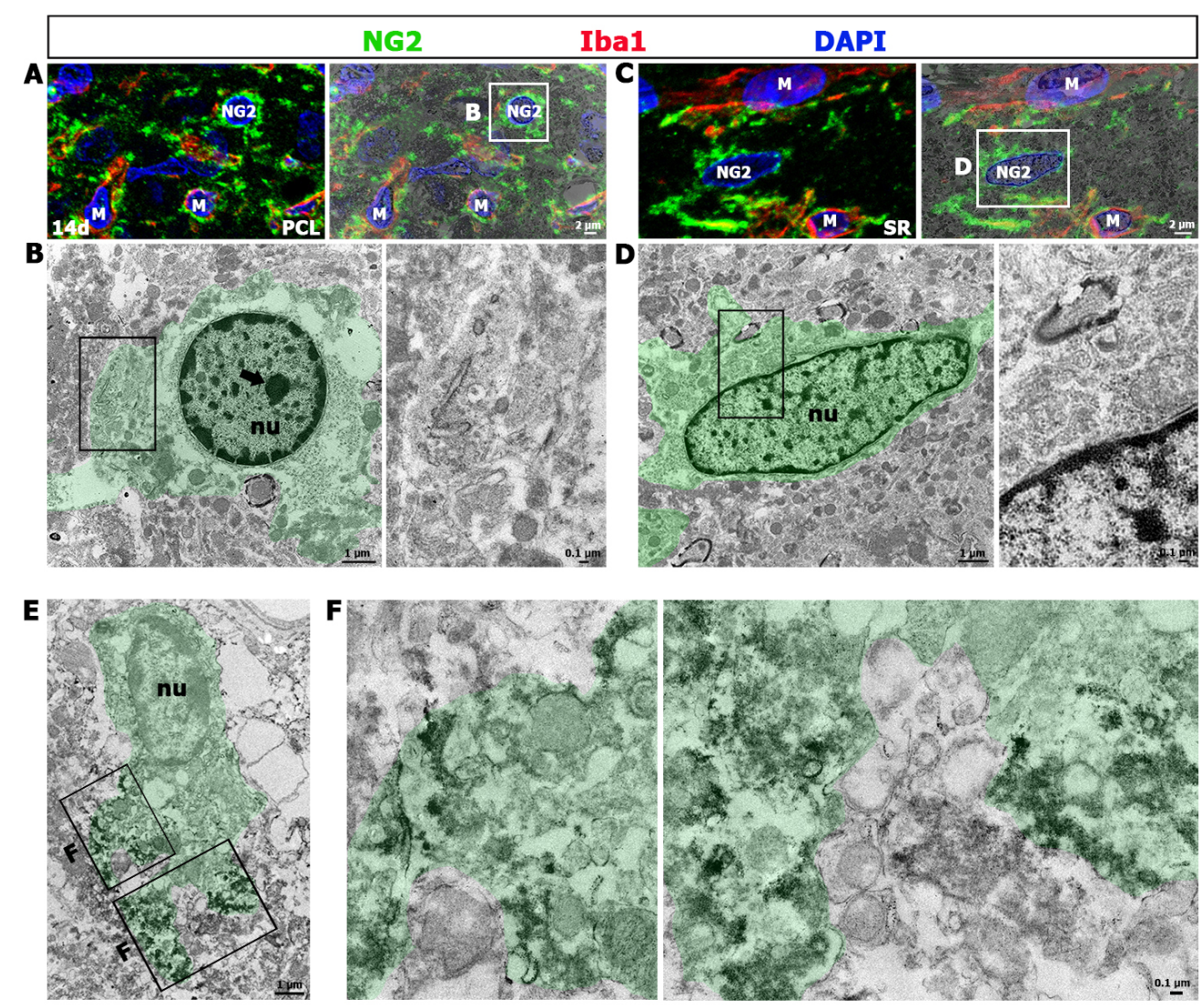

Fig. 10. Representative correlative light and electron microscopic images and pre-embedding immunoelectron microscopic images showing the ultrastructural characterization of reactive NG2 glia in the hippocampal CA1 region on day 14 after reperfusion. Confocal microscopic images of semithin sections double-labeled for NG2 and Ibal (left panels of A and C), images of confocal microscopic data overlaid onto the corresponding electron microscopic images (right panels in A and C), and the corresponding transmission electron microscopic images obtained from the boxed areas in right panels in A and C (left panels of B and D). Moreover, the boxed areas in the left panels in B and D are enlarged in the right panels of B and D, respectively. Note that reactive NG2 glia (NG2; shaded in green) have euchromatic nuclei (labelled as nu in the left panels of B and D) with prominent nucleoli (arrow in the left panel of B) and cytoplasm containing a well-developed rough endoplasmic reticulum. Cell nuclei are stained with DAPI. (E, F) Pre-embedding immunoelectron microscopic images of reactive NG2 glia in the stratum radiatum. The boxed areas in E are enlarged in F. Note that NG2 immunoreactivity, as indicated by highly electron-dense DAB grains, is localized along the plasma membrane of NG2 glia (shaded in green) and the adjacent extracellular matrix. Scale bars represent: $2 \mu \mathrm{m}$ in A and C; $1 \mu \mathrm{m}$ in the left panels in B, D, and E; and $0.1 \mu \mathrm{m}$ in the right panels in B, D and F.

NG2 glia in areas distant from the vasculature were antigenically distinct from astrocytes, mature oligodendrocytes, and microglia, all of which were devoid of specific NG2 immunoreactivity (Fig. 3). In the ischemic hippocampus, NG2 expression was induced in activated microglia/macrophages, with neither reactive astrocytes nor oligodendrocytes exhibiting specific NG2 immunoreactivity (Fig. 5). In addition, NG2 glia and pericytes could be easily differentiated based on their branched versus crescent-shaped morphology, respectively [49]. Thus, our data clearly indicate that NG2-positive cells in the ischemic CA1 hippocampus were comprised of cells with constitutive (NG2 glia) and ischemia-induced (activated microglia/macrophages) expression of NG2.

We found that in the CA1 hippocampus of sham-operated controls, NG2 glia exhibited morphological features typical of resting
NG2 with small cell bodies from which multiple fine processes radiate in all directions, as described previously [16, 44, 50-52]. In addition, our data using a correlative light and electron microscopic approach revealed that these resting NG2 glia had scanty cytoplasm with few cytoplasmic organelles. Resting NG2 glia shared morphological and ultrastructural features in the two strata of the CA1 hippocampus. Activation of NG2 glia was evident in both the pyramidal cell layer and the stratum radiatum of the CA1 hippocampus starting within 3 days after reperfusion, which correlates with the spatiotemporal profiles of neurodegeneration after ischemic injury [27, 53-55], and their activation was sustained for at least 28 days after reperfusion. In the ischemic CA 1 hippocampus, NG2 glia underwent proliferation and dynamic morphological changes, which were very similar to NG2 glia be- 
coming reactive in response to a variety of insults $[8,12-14,16,34$, 44]. Besides, reactive NG2 glia had abundant rough endoplasmic reticulum and well-developed Golgi complexes compared to resting NG2 glia, consistent with the findings of our previous study [16]. Thus, our data indicate that activation of NG2 glia is a general phenomenon during CNS insults, rather than being limited to a specific CNS region or to a particular experimental model. Moreover, we found that proliferation and morphological changes of NG2 glia occurred in a distinct time- and layer-specific manner in the ischemic CA1 hippocampus. In particular, fluorescent images acquired and rendered as 3D reconstruction provided evidence for the differences in cellular morphology and arborization of individual NG2 glia in both examined strata of the CA1 hippocampus during the post-ischemic period despite the morphological similarities of resting NG2 glia in these strata. Reactive NG2 glia in the pyramidal cell layer had shortened, scarcely branched processes and generally maintained these features overall during the post-ischemic period. By contrast, reactive NG2 glia in the stratum radiatum exhibited multiple and complex branching processes after reperfusion, and at 7 14 days after reperfusion, the morphology and arborization status of the NG2 glia processes could not be discerned because amorphous clouds of NG2 staining surrounded these cells. This cloudy appearance of reactive NG2 glia was also described in the denervated outer molecular layer of the hippocampal dentate gyrus after entorhinal cortex lesion, suggesting that this cloudy immunostaining is, at least in part, caused by the secretion of NG2 into the extracellular matrix [51]. In line with this, the localization of accumulated NG2 appears rather diffuse and has a lack of distinct cellular structure in the spinal cord during experimental autoimmune encephalomyelitis, indicating that NG2 is secreted and incorporated into the extracellular matrix [56]. In particular, our pre-embedding immunoelectron microscopic findings demonstrated that NG2 immunoreactivity was specifically associated with the plasma membrane and its adjacent extracellular matrix of NG2 glia in the stratum radiatum 14 days following reperfusion (Fig. 10), as previously reported [51], raising the possibility that NG2 is secreted into the extracellular matrix by NG2 glia. In addition, the NG2 protein undergoes cleavage of its ectodomain, and the shedded form of NG2 is increased in the injured CNS [57]. Thus, it is likely that NG2 production actively proceeds in reactive NG2 glia of the stratum radiatum at 7 14 days after reperfusion and that these NG2 glia represent the most active phenotype in response to tissue degeneration.

It is well-known that extensive neuronal death and dendritic damage occur in the CA1 hippocampal region, commencing 2 to 3 days after transient forebrain ischemia and reaching their maximal effects within $1 \sim 2$ weeks $[27,53,58]$. Furthermore, re- generative processes such as neurite growth are lacking in the CA1 hippocampus 15 days after ischemia [59]. Thus, it is likely that the temporal pattern of reactive NG2 glia phenotypes in the stratum radiatum overlaps with the period of ongoing dendrite degeneration observed in pyramidal neurons, resulting from ischemic insults to this layer. In this sense, it is interesting that although the numbers of NG2 glia in both strata were significantly increased on day 3 after reperfusion and elevated until day 28 , their temporal profiles were different in these strata. The number of NG2 glia in the pyramidal cell layer peaked at day 3 and was not changed significantly throughout the injury period, whereas NG2 glia in the stratum radiatum showed a tendency to increase in number with time. This increase was attributed to enhanced proliferation of NG2 glia, as determined using triple-labeling for NG2, Iba1, and Ki67. The proliferation of NG2 glia in both strata was most prominent at day 3 and was considerably reduced by day 7 . However, a more sustained proliferation of NG2 glia could be observed in the stratum radiatum, relative to that in the pyramidal cell layer, suggesting that this may be a response to ongoing degeneration in this layer. Given that NG2 glia have direct synaptic contacts with neurons in multiple brain regions, including the hippocampus [4, 60-64], our data suggest a possible link between the activation of NG2 glia and significant damage to dendrites, which may have direct synaptic contacts with NG2 glia. However, whether the loss of functional synapses in NG2 glia results in the activation of NG2 glia, and what triggers the activation of NG2 glia after ischemic insults, remains to be addressed.

The functional significance of reactive NG2 glia in the injured CNS is not fully understood. In particular, the role of NG2 with respect to axonal growth is controversial. Chondroitin sulfate proteoglycans including NG2 are widely accepted to be inhibitory to axonal regeneration; NG2 glia inhibit axonal regeneration after CNS lesions, and their targeted reduction can improve regeneration $[18,65-70]$. Conversely, NG2 is thought to facilitate axonal growth and stabilize the axon during development and regeneration [18, 71-75]. In addition, NG2 upregulation in the denervated hippocampal dentate gyrus coincides with the sprouting response, suggesting that NG2 regulates axonal growth [51]. Furthermore, ablation of NG2 glia produces defects in hippocampal neurons due to excessive neuroinflammation, suggesting that NG2 glia maintain neuronal functions and survival via the control of neuroimmunological functions [76]. Thus, it can be speculated that long-lasting and selective induction of NG2 in areas affected by ischemia is related not only to the neurodegeneration but possibly to subsequent neuronal reorganization as well. However, the functional significance of reactive NG2 glia was not fully clarified in the present study; thus, further studies are required to determine 
the precise role and mechanisms of NG2 glia in the ischemic brain, and also whether layer-specific phenotypes of NG2 glia indeed reflect their potential functions or simply the characteristic cytoarchitectonic features of these two layers.

Another interesting finding was that induced NG2 expression in activated microglia/macrophages exhibited a characteristic strata-dependent pattern in the ischemic CA1 hippocampus. NG2 induction was prominent in macrophage-like phenotypes located predominantly in the pyramidal cell layer; this is in comparison to the activated stellate microglial cells observed in the stratum radiatum. This is in agreement with previous reports showing that activated microglia and macrophages within the pyramidal cell layer cluster around degenerating neurons, while rod-shaped reactive microglia are observed alongside the degenerating pyramidal dendrites in the stratum radiatum, which was associated with dendritic degeneration [31-33,77]. Despite substantial information regarding the induction of NG2 in activated microglia/macrophages after CNS insults, its specific roles are still unknown. Moransard et al. [56] reported that NG2 deficiency has no effect in experimental autoimmune encephalomyelitis, indicating that NG2 does not play an important role in macrophage/microglia functions in autoimmunity. However, there are several suggestions regarding the potential roles of NG2 induction in activated microglia/macrophages. NG2 regulates the production of inflammatory cytokines and neuroprotective factors in activated microglia/macrophages $[22,78]$. In fact, the induction of NG2 expression in these cells leads to microglial activation or alteration of functional activities, such as phagocytosis [22, 24, 45, 78]. In addition, NG2 expression increases the invasive and migratory capabilities of glioma cells by facilitating interactions with extracellular matrix proteins [79, 80]. Interestingly, we found that NG2 expression was strongly induced in macrophage-like phenotypes with amoeboid morphology rather than in activated microglia with evident processes, which was confirmed by quantification of the mean fluorescence intensity of NG2 expression in these cells (Fig. 7). Thus, our data raise the possibility that NG2 expression in brain macrophages may reflect the activation/phagocytosis of microglia or infiltrating blood-borne macrophages, as suggested previously $[22,24,45,78]$, although the exact functional significance remains to be elucidated. We cannot exclude the possibility that this observation was simply related to the functional status of microglia and macrophages.

In summary, our data demonstrate that ischemic insults induced the transformation of NG2 glia into distinct reactive phenotypes in the pyramidal cell layer and the stratum radiatum of the CA1 hippocampus. In addition, NG2 expression was induced after ischemic insults in activated microglia/macrophages in the CA1 hippocampus in a layer-specific manner. Thus, these findings in- dicate that activation of NG2 glia and induced NG2 expression in activated microglia/macrophages occur in a characteristic timeand layer-specific manner in the ischemic CA1 hippocampus. These characteristic profiles of reactive NG2 glia could be secondary to the degeneration processes occurring in the cell bodies or dendritic domains of hippocampal CA1 pyramidal neurons after ischemic insults.

\section{DATA AVAILABILITY}

The datasets used and/or analyzed during the current study are available from the corresponding author on reasonable request.

\section{AUTHOR CONTRIBUTIONS}

All authors have contributed significantly to the research and the article preparation: XJ contributed to the treatment of animals, immunohistochemistry, immunoelectron microscopy, quantitative and qualitative image analysis. T-RR and SK contributed to the treatment of animals and immunohistochemistry. HK worked on the electron microscopy. M-YL worked on the design of the study, data analysis and final manuscript preparation.

\section{FUNDING}

This research was supported by the grants from the National Research Foundation of Korea (NRF) [grant number NRF2017R1A2B4002922).

\section{CONFLICT OF INTEREST}

The authors report no conflict of interest with any person or Institute.

\section{ETHICAL APPROVAL}

All interventions and animal care provisions were in accordance with the Laboratory Animals Welfare Act, the Guide for the Care and Use of Laboratory Animals, and the Guidelines and Policies for Rodent Survival Surgery provided by the IACUC (Institutional Animal Care and Use Committee) at the College of Medicine, The Catholic University of Korea (Approval number: CUMS-20170321-05).

\section{REFERENCES}

1. Xu JP, Zhao J, Li S (2011) Roles of NG2 glial cells in diseases 
of the central nervous system. Neurosci Bull 27:413-421.

2. Küspert M, Wegner M (2016) SomethiNG 2 talk abouttranscriptional regulation in embryonic and adult oligodendrocyte precursors. Brain Res 1638:167-182.

3. Nishiyama A, Komitova M, Suzuki R, Zhu X (2009) Polydendrocytes (NG2 cells): multifunctional cells with lineage plasticity. Nat Rev Neurosci 10:9-22.

4. Dimou L, Gallo V (2015) NG2-glia and their functions in the central nervous system. Glia 63:1429-1451.

5. Eugenín-von Bernhardi J, Dimou L (2016) NG2-glia, more than progenitor cells. Adv Exp Med Biol 949:27-45.

6. Viganò F, Dimou L (2016) The heterogeneous nature of NG2glia. Brain Res 1638:129-137.

7. Levine JM, Reynolds R (1999) Activation and proliferation of endogenous oligodendrocyte precursor cells during ethidium bromide-induced demyelination. Exp Neurol 160:333-347.

8. McTigue DM, Wei P, Stokes BT (2001) Proliferation of NG2positive cells and altered oligodendrocyte numbers in the contused rat spinal cord. J Neurosci 21:3392-3400.

9. Kang SH, Fukaya M, Yang JK, Rothstein JD, Bergles DE (2010) $\mathrm{NG} 2+\mathrm{CNS}$ glial progenitors remain committed to the oligodendrocyte lineage in postnatal life and following neurodegeneration. Neuron 68:668-681.

10. Guo F, Maeda Y, Ma J, Delgado M, Sohn J, Miers L, Ko EM, Bannerman P, Xu J, Wang Y, Zhou C, Takebayashi H, Pleasure D (2011) Macroglial plasticity and the origins of reactive astroglia in experimental autoimmune encephalomyelitis. J Neurosci 31:11914-11928.

11. Tripathi RB, Rivers LE, Young KM, Jamen F, Richardson WD (2010) NG2 glia generate new oligodendrocytes but few astrocytes in a murine experimental autoimmune encephalomyelitis model of demyelinating disease. J Neurosci 30:16383-16390.

12. Dimou L, Simon C, Kirchhoff F, Takebayashi H, Götz M (2008) Progeny of Olig2-expressing progenitors in the gray and white matter of the adult mouse cerebral cortex. J Neurosci 28:10434-10442.

13. Simon C, Götz M, Dimou L (2011) Progenitors in the adult cerebral cortex: cell cycle properties and regulation by physiological stimuli and injury. Glia 59:869-881.

14. Zhang R, Chopp M, Zhang ZG (2013) Oligodendrogenesis after cerebral ischemia. Front Cell Neurosci 7:201.

15. Anderova M, Vorisek I, Pivonkova H, Benesova J, Vargova L, Cicanic M, Chvatal A, Sykova E (2011) Cell death/proliferation and alterations in glial morphology contribute to changes in diffusivity in the rat hippocampus after hypoxiaischemia. J Cereb Blood Flow Metab 31:894-907.
16. Jin X, Riew TR, Kim HL, Choi JH, Lee MY (2018) Morphological characterization of NG2 glia and their association with neuroglial cells in the 3-nitropropionic acid-lesioned striatum of rat. Sci Rep 8:5942.

17. Bu J, Akhtar N, Nishiyama A (2001) Transient expression of the NG2 proteoglycan by a subpopulation of activated macrophages in an excitotoxic hippocampal lesion. Glia 34:296310.

18. Jones LL, Yamaguchi Y, Stallcup WB, Tuszynski MH (2002) NG2 is a major chondroitin sulfate proteoglycan produced after spinal cord injury and is expressed by macrophages and oligodendrocyte progenitors. J Neurosci 22:2792-2803.

19. Yokoyama A, Sakamoto A, Kameda K, Imai Y, Tanaka J (2006) NG2 proteoglycan-expressing microglia as multipotent neural progenitors in normal and pathologic brains. Glia 53:754768.

20. Fiedorowicz A, Figiel I, Zaremba M, Dzwonek K, OderfeldNowak B (2008) The ameboid phenotype of NG2 (+) cells in the region of apoptotic dentate granule neurons in trimethyltin intoxicated mice shares antigen properties with microglia/ macrophages. Glia 56:209-222.

21. Matsumoto H, Kumon Y, Watanabe H, Ohnishi T, Shudou M, Chuai M, Imai Y, Takahashi H, Tanaka J (2008) Accumulation of macrophage-like cells expressing NG2 proteoglycan and Ibal in ischemic core of rat brain after transient middle cerebral artery occlusion. J Cereb Blood Flow Metab 28:149-163.

22. Smirkin A, Matsumoto H, Takahashi H, Inoue A, Tagawa M, Ohue S, Watanabe H, Yano H, Kumon Y, Ohnishi T, Tanaka J (2010) Iba1(+)/NG2(+) macrophage-like cells expressing a variety of neuroprotective factors ameliorate ischemic damage of the brain. J Cereb Blood Flow Metab 30:603-615.

23. Zhu X, Hill RA, Dietrich D, Komitova M, Suzuki R, Nishiyama A (2011) Age-dependent fate and lineage restriction of single NG2 cells. Development 138:745-753.

24. Zhu L, Xiang P, Guo K, Wang A, Lu J, Tay SS, Jiang H, He BP (2012) Microglia/monocytes with NG2 expression have no phagocytic function in the cortex after LPS focal injection into the rat brain. Glia 60:1417-1426.

25. Ozerdem U, Grako KA, Dahlin-Huppe K, Monosov E, Stallcup WB (2001) NG2 proteoglycan is expressed exclusively by mural cells during vascular morphogenesis. Dev Dyn 222:218-227.

26. Ito U, Spatz M, Walker JT Jr, Klatzo I (1975) Experimental cerebral ischemia in mongolian gerbils. I. light microscopic observations. Acta Neuropathol 32:209-223.

27. Kirino T, Sano K (1984) Selective vulnerability in the gerbil hippocampus following transient ischemia. Acta Neuropathol 
62:201-208.

28. Pulsinelli WA (1985) Selective neuronal vulnerability: morphological and molecular characteristics. Prog Brain Res 63:29-37.

29. Joe EH, Choi DJ, An J, Eun JH, Jou I, Park S (2018) Astrocytes, microglia, and Parkinson's disease. Exp Neurobiol 27:77-87.

30. Cho SB, Eum WS, Shin MJ, Kwon HJ, Park JH, Choi YJ, Park J, Han KH, Kang JH, Kim DS, Cho SW, Kim DW, Choi SY (2019) Transduced Tat-aldose reductase protects hippocampal neuronal cells against oxidative stress-induced damage. Exp Neurobiol 28:612-627.

31. Jørgensen MB, Finsen BR, Jensen MB, Castellano B, Diemer NH, Zimmer J (1993) Microglial and astroglial reactions to ischemic and kainic acid-induced lesions of the adult rat hippocampus. Exp Neurol 120:70-88.

32. Kettenmann H, Hanisch UK, Noda M, Verkhratsky A (2011) Physiology of microglia. Physiol Rev 91:461-553.

33. Sugawara T, Lewén A, Noshita N, Gasche Y, Chan PH (2002) Effects of global ischemia duration on neuronal, astroglial, oligodendroglial, and microglial reactions in the vulnerable hippocampal CA1 subregion in rats. J Neurotrauma 19:8598.

34. Ong WY, Levine JM (1999) A light and electron microscopic study of NG2 chondroitin sulfate proteoglycan-positive oligodendrocyte precursor cells in the normal and kainatelesioned rat hippocampus. Neuroscience 92:83-95.

35. Pulsinelli WA, Brierley JB (1979) A new model of bilateral hemispheric ischemia in the unanesthetized rat. Stroke 10:267-272.

36. Lee MY, Choi YS, Choi JS, Min DS, Chun MH, Kim ON, Lee SB, Kim SY (2002) An immunohistochemical study of APG2 protein in the rat hippocampus after transient forebrain ischemia. Brain Res 924:237-241.

37. Paxinos G, Watson C (2006) The rat brain in stereotaxic coordinates. 6th ed. Academic Press, New York.

38. Nitatori T, Sato N, Waguri S, Karasawa Y, Araki H, Shibanai K, Kominami E, Uchiyama Y (1995) Delayed neuronal death in the CA1 pyramidal cell layer of the gerbil hippocampus following transient ischemia is apoptosis. J Neurosci 15:10011011.

39. Niimura M, Takagi N, Takagi K, Funakoshi H, Nakamura T, Takeo S (2006) Effects of hepatocyte growth factor on phosphorylation of extracellular signal-regulated kinase and hippocampal cell death in rats with transient forebrain ischemia. Eur J Pharmacol 535:114-124.

40. Matesic DF, Lin RC (1994) Microtubule-associated protein 2 as an early indicator of ischemia-induced neurodegeneration in the gerbil forebrain. J Neurochem 63:1012-1020.

41. Lin B, Ginsberg MD, Busto R (1998) Hyperglycemic exacerbation of neuronal damage following forebrain ischemia: microglial, astrocytic and endothelial alterations. Acta Neuropathol 96:610-620.

42. Hampton DW, Rhodes KE, Zhao C, Franklin RJ, Fawcett JW (2004) The responses of oligodendrocyte precursor cells, astrocytes and microglia to a cortical stab injury, in the brain. Neuroscience 127:813-820.

43. Casella GT, Bunge MB, Wood PM (2004) Improved immunocytochemical identification of neural, endothelial, and inflammatory cell types in paraffin-embedded injured adult rat spinal cord. J Neurosci Methods 139:1-11.

44. Nishiyama A, Yu M, Drazba JA, Tuohy VK (1997) Normal and reactive NG2+ glial cells are distinct from resting and activated microglia. J Neurosci Res 48:299-312.

45. Xiang P, Zhu L, Jiang H, He BP (2015) The activation of NG2 expressing cells is downstream to microglial reaction and mediated by the transforming growth factor beta 1. J Neuroimmunol 279:50-63.

46. Gerdes J, Schwab U, Lemke H, Stein H (1983) Production of a mouse monoclonal antibody reactive with a human nuclear antigen associated with cell proliferation. Int J Cancer 31:1320.

47. Scholzen T, Gerdes J (2000) The Ki-67 protein: from the known and the unknown. J Cell Physiol 182:311-322.

48. Stallcup WB (2002) The NG2 proteoglycan: past insights and future prospects. J Neurocytol 31:423-435.

49. Hesp ZC, Yoseph RY, Suzuki R, Jukkola P, Wilson C, Nishiyama A, McTigue DM (2018) Proliferating NG2-cell-dependent angiogenesis and scar formation alter axon growth and functional recovery after spinal cord injury in mice. J Neurosci 38:1366-1382.

50. Dawson MR, Polito A, Levine JM, Reynolds R (2003) NG2expressing glial progenitor cells: an abundant and widespread population of cycling cells in the adult rat CNS. Mol Cell Neurosci 24:476-488.

51. Dehn D, Burbach GJ, Schäfer R, Deller T (2006) NG2 upregulation in the denervated rat fascia dentata following unilateral entorhinal cortex lesion. Glia 53:491-500.

52. Kurkowska-Jastrzebska I, Zaremba M, Członkowska A, Oderfeld-Nowak B (2010) Down-regulation of microglia and NG2-positive cells reaction in trimethyltin-injured hippocampus of rats treated with myelin basic protein-reactive $\mathrm{T}$ cells: possible contribution to the neuroprotective effect of $\mathrm{T}$ cells. J Neurosci Res 88:24-32.

53. Pulsinelli WA, Brierley JB, Plum F (1982) Temporal profile of 
neuronal damage in a model of transient forebrain ischemia. Ann Neurol 11:491-498.

54. Petito CK, Kraig RP, Pulsinelli WA (1987) Light and electron microscopic evaluation of hydrogen ion-induced brain necrosis. J Cereb Blood Flow Metab 7:625-632.

55. Fryd Johansen F, Balslev Jørgensen M, Diemer NH (1983) Resistance of hippocampal CA- 1 interneurons to $20 \mathrm{~min}$ of transient cerebral ischemia in the rat. Acta Neuropathol 1983;61:135-140.

56. Moransard M, Dann A, Staszewski O, Fontana A, Prinz M, Suter T (2011) NG2 expressed by macrophages and oligodendrocyte precursor cells is dispensable in experimental autoimmune encephalomyelitis. Brain 134:1315-1330.

57. Asher RA, Morgenstern DA, Properzi F, Nishiyama A, Levine JM, Fawcett JW (2005) Two separate metalloproteinase activities are responsible for the shedding and processing of the NG2 proteoglycan in vitro. Mol Cell Neurosci 29:82-96.

58. Smith ML, Auer RN, Siesjö BK (1984) The density and distribution of ischemic brain injury in the rat following 2-10 min of forebrain ischemia. Acta Neuropathol 64:319-332.

59. Yoo DY, Kwon HJ, Lee KY, Jung HY, Kim JW, Park JH, Choi JH, Yoon YS, Won MH, Kim DW, Hwang IK (2016) Lack of evidence in neurite growth in the gerbil hippocampal CA1 region 15 days after transient forebrain ischemia. Anim Cells Syst 20:237-245.

60. Bergles DE, Roberts JD, Somogyi P, Jahr CE (2000) Glutamatergic synapses on oligodendrocyte precursor cells in the hippocampus. Nature 405:187-191.

61. Lin SC, Huck JH, Roberts JD, Macklin WB, Somogyi P, Bergles DE (2005) Climbing fiber innervation of NG2-expressing glia in the mammalian cerebellum. Neuron 46:773-785.

62. Paukert M, Bergles DE (2006) Synaptic communication between neurons and NG2+ cells. Curr Opin Neurobiol 16:515521.

63. Trotter J, Karram K, Nishiyama A (2010) NG2 cells: properties, progeny and origin. Brain Res Rev 63:72-82.

64. De Biase LM, Nishiyama A, Bergles DE (2010) Excitability and synaptic communication within the oligodendrocyte lineage. J Neurosci 30:3600-3611.

65. Fidler PS, Schuette K, Asher RA, Dobbertin A, Thornton SR, Calle-Patino Y, Muir E, Levine JM, Geller HM, Rogers JH, Faissner A, Fawcett JW (1999) Comparing astrocytic cell lines that are inhibitory or permissive for axon growth: the major axon-inhibitory proteoglycan is NG2. J Neurosci 19:87788788.

66. Levine JM, Reynolds R, Fawcett JW (2001) The oligodendrocyte precursor cell in health and disease. Trends Neurosci
24:39-47.

67. Siebert JR, Conta Steencken A, Osterhout DJ (2014) Chondroitin sulfate proteoglycans in the nervous system: inhibitors to repair. Biomed Res Int 2014:845323.

68. Zhang F, Clarke JD, Santos-Ruiz L, Ferretti P (2002) Differential regulation of fibroblast growth factor receptors in the regenerating amphibian spinal cord in vivo. Neuroscience 114:837-848.

69. Sandvig A, Berry M, Barrett LB, Butt A, Logan A (2004) Myelin-, reactive glia-, and scar-derived CNS axon growth inhibitors: expression, receptor signaling, and correlation with axon regeneration. Glia 46:225-251.

70. Lau BY, Fogerson SM, Walsh RB, Morgan JR (2013) Cyclic AMP promotes axon regeneration, lesion repair and neuronal survival in lampreys after spinal cord injury. Exp Neurol 250:31-42

71. Yang Z, Suzuki R, Daniels SB, Brunquell CB, Sala CJ, Nishiyama A (2006) NG2 glial cells provide a favorable substrate for growing axons. J Neurosci 26:3829-3839.

72. Busch SA, Horn KP, Cuascut FX, Hawthorne AL, Bai L, Miller RH, Silver J (2010) Adult NG2+ cells are permissive to neurite outgrowth and stabilize sensory axons during macrophageinduced axonal dieback after spinal cord injury. J Neurosci 30:255-265.

73. Jones LL, Sajed D, Tuszynski MH (2003) Axonal regeneration through regions of chondroitin sulfate proteoglycan deposition after spinal cord injury: a balance of permissiveness and inhibition. J Neurosci 23:9276-9288.

74. Hossain-Ibrahim MK, Rezajooi K, Stallcup WB, Lieberman AR, Anderson PN (2007) Analysis of axonal regeneration in the central and peripheral nervous systems of the NG2deficient mouse. BMC Neurosci 8:80.

75. O'Shea TM, Burda JE, Sofroniew MV (2017) Cell biology of spinal cord injury and repair. J Clin Invest 127:3259-3270.

76. Nakano M, Tamura Y, Yamato M, Kume S, Eguchi A, Takata K, Watanabe Y, Kataoka Y (2017) NG2 glial cells regulate neuroimmunological responses to maintain neuronal function and survival. Sci Rep 7:42041.

77. Lambertsen KL, Deierborg T, Gregersen R, Clausen BH, Wirenfeldt M, Nielsen HH, Dalmau I, Diemer NH, DagnaesHansen F, Johansen FF, Keating A, Finsen B (2011) Differences in origin of reactive microglia in bone marrow chimeric mouse and rat after transient global ischemia. J Neuropathol Exp Neurol 70:481-494.

78. Gao Q, Lu J, Huo Y, Baby N, Ling EA, Dheen ST (2010) NG2, a member of chondroitin sulfate proteoglycans family mediates the inflammatory response of activated microglia. Neurosci- 
ence 165:386-394.

79. Burg MA, Tillet E, Timpl R, Stallcup WB (1996) Binding of the NG2 proteoglycan to type VI collagen and other extracel- lular matrix molecules. J Biol Chem 271:26110-26116.

80. Yadavilli S, Hwang EI, Packer RJ, Nazarian J (2016) The role of NG2 proteoglycan in glioma. Transl Oncol 9:57-63. 